\title{
Photometric studies of some starburst galaxies
}

\author{
A. Chitre and U.C. Joshi \\ Physical Research Laboratory, Navrangpura, Ahmedabad, Gujarat 380 009, India \\ e-mail: chitre@prl.ernet.in
}

Received May 27, 1998; accepted June 14, 1999

\begin{abstract}
We present the results of a detailed morphological analysis of ten starburst galaxies selected from the Markarian catalog of uv-excess objects. CCD surface photometry of these galaxies was carried out based on observations made in $B, V$ (Johnson) and $R, I$ (Kron-Cousins) band passes. We present the radial variations of surface brightness, ellipticity, position angle and the colour indices for each galaxy obtained using ellipse fitting isophotal analysis. The residual images constructed for extracting the fine structure are also presented. A variety of morphological types are found to host the starburst phenomenon. The star formation activity is not confined to the nuclear region alone, but it also occurs at various locations in the galaxy and is seen as clumpy regions. The colour index and the residual images are used for deriving information about the sites of enhanced star formation activity and the triggers of the starburst. The luminosity profiles show an exponential behaviour in the outer region. The disk scale lengths and the half-light radii are derived. The contribution of the burst component has been estimated and the colours of the burst component are presented. Strong isophotal twisting is detected in all the S0 and E galaxies: Mrk 1002, Mrk 1308 and Mrk 14, in the sample. This is accompanied by boxiness in some cases, suggesting that a merger is responsible for the starburst activity in these galaxies. In case of isolated spirals, a bar or a central oval distortion appear to be the likely trigger for the starburst.
\end{abstract}

Key words: galaxies: starburst — galaxies: photometry galaxies: stucture

\section{Introduction}

The term "starburst" was first coined by Weedman (1973) to describe galaxies experiencing episodes of star formation that are too intense to be sustained over the

Send offprint requests to: A. Chitre lifetime of the galaxy. These bursts of star formation produce $10^{7}-10^{9} M_{\odot}$ of OB stars (Balzano 1983) which are the dominant source of the galaxy's luminosity. Since OB stars emit copiously in the ultraviolet, many starburst galaxies were detected in Markarian et al. (1979) survey of uv-excess objects and references therein. These lists form one of the largest databases of optically selected starburst galaxies. Huchra (1977) conducted a detailed aperture photometric study of a large number of these galaxies. A spectrophotometric survey was conducted by Balzano (1983) wherein she studied the spectra of the nuclear regions in the starburst galaxies. Larson \& Tinsley (1978) were the first to put forward the idea that "bursts of star formation" could be triggered by tidal forces in interacting galaxies. Combes et al. (1990) showed that in the presence of non-axisymmetric potentials like bars or companions, gravitational torques are capable of driving the gas in a galaxy into the central regions. This gas may pile-up at the ILR, if it is present or proceed right up to the nuclear region where it may fragment to form ring-shaped or nuclear starbursts. Interactions may also induce extranuclear starbursts as is seen in the $\mathrm{H} \alpha$ imaging study of Garcia-Barreto et al. (1996).

Dynamical disturbances experienced by the galaxy are reflected in the morphology of the galaxy in the form of peculiar structures. Tidal tails, bridges, asymmetric outer envelopes are some manifestations of the perturbing processes (Combes 1987 and references therein). These disturbances are possible triggers of the starburst phenomenon. Interactions, mergers and the presence of a bar were proposed as the most likely triggers for the starburst. However, it has been observed that a few starburst galaxies show neither visible signs of disturbances in the form of peculiar morphologies nor the presence of a bar in their direct images. A detailed study of the underlying galaxy is especially important in such cases to probe the cause of the starburst. An inspection of the Markarian sample of starburst galaxies reveals that though the starburst phenomenon is predominantly found in spiral galaxies, it is not confined to spirals alone and the sample contains a 
Table 1. Sample of starburst galaxies

\begin{tabular}{llllllllllll}
\hline GALAXY & $\alpha$ & $\delta$ & TYPE $^{\text {a }}$ & \multicolumn{2}{c}{ Distance } & \multicolumn{2}{c}{ exposure time (minutes) } & ellipticity & inc & PA \\
& $(2000)$ & $(2000)$ & & Mpc & $B$ & $V$ & $R$ & $I$ & & & ${ }^{\circ}$ \\
\hline Mrk 14 & $08: 10: 59.1$ & $72: 47: 41$ & S0? & 42 & 23 & 11 & 8 & 10 & 0.2 & 41 & 165 \\
Mrk 87 & $08: 21: 41.2$ & $73: 59: 23$ & SB0/a & 37 & 50 & 6 & 6 & 6 & 0.5 & 65.5 & 60 \\
Mrk 213 & $12: 31: 20.5$ & $57: 57: 47$ & SBa & 42 & 25 & 12 & 11 & 11 & 0.6 & 72.5 & 141 \\
Mrk 363 & $01: 50: 58.0$ & $21: 59: 50$ & Scp & 39 & 17 & 6 & 6 & 5.3 & 0.25 & 45.5 & 171 \\
Mrk 449 & $13: 11: 30.8$ & $36: 16: 52$ & Sap & 15 & 15 & 7.5 & 7.5 & 10 & 0.7 & 80 & 82 \\
Mrk 743 & $11: 38: 12.9$ & $12: 06: 43$ & E0p & 13 & 13.3 & 7.5 & 7 & 6 & - & - & - \\
Mrk 781 & $12: 53: 50.7$ & $09: 42: 33$ & SBc & 37 & 13.3 & 10 & 8.6 & 6 & 0.1 & 30 & 35 \\
Mrk 1002 & $01: 37: 17.5$ & $05: 52: 38$ & E1 & 42 & 20 & 9.16 & 5 & 5 & 0.26 & 46 & 163 \\
Mrk 1308 & $11: 54: 12.2$ & $00: 08: 11$ & S0 & 14 & 13.3 & 7.5 & 6 & 8 & 0.1 & 30 & 20 \\
Mrk 1379 & $14: 17: 40.3$ & $-07: 25: 03$ & SBbc dbl & 37 & 16.6 & 6 & 6 & 8.6 & 0.45 & 61.5 & 51 \\
\hline
\end{tabular}

a Markarian catalogue (downloaded from CDS).

large number of S0 and elliptical galaxies, in addition to the ones that show too peculiar a morphology to be classified properly. A morphological study of a sample of starburst galaxies through several spectral bands is expected to help shed some light on the conditions leading to the onset of a starburst and the environment of the underlying galaxies. The general structure of the underlying galaxy can be studied through techniques like ellipse fitting. This technique is especially useful in studying isophotal twists, the presence of bars, rings, shells, etc. in the galaxy. In recent years, studies conducted by Wozniak et al. (1995) and Jungwiert et al. (1997) on samples of spiral galaxies using this technique have been useful in detecting underlying structures like oval distortions, shells, rings and hitherto undetected bars. This technique can be used to study the starburst galaxies in a similar manner. The fine structure extracted from the isophotal analysis can be interpreted to give valuable information about the underlying galaxy and the nature of the disturbances experienced by it (Kenney et al. 1996). In the present study, a sample of starburst galaxies was observed in $B V R I$ bands and we present results on ten galaxies.

\section{Observations and data reduction}

The sample presented in this paper is a part of a sample of starburst galaxies selected from the Markarian catalog. Galaxies with $m_{v}$ brighter than $14^{\mathrm{m}} 5$ and angular sizes greater than $30^{\prime \prime}$ were selected. No consideration was made for any particular morphological type and the sample is unbiased towards the morphology of the galaxy. In this paper, results based on the photometric studies of ten galaxies from the sample are presented. These ten galaxies are listed in Table 1, along with their coordinates, morphological types, distances $\left(H_{0}=75 \mathrm{~km} \mathrm{~s}^{-1} \mathrm{Mpc}^{-1}\right.$ has been adopted throughout), total exposure times in each band, the derived ellipticity, inclination and position angle.

The observations were made at the Cassegrain focus of the $1.2 \mathrm{~m}$ Gurushikhar telescope employing a thinned back illuminated Tektronix $1024 \times 1024$ CCD chip. The observations were carried out under photometric conditions with typical seeing of $1.5^{\prime \prime}$. Binning of $2 \times 2$ was employed before recording the images to increase the signal-to-noise ratio of the measurements and keeping in mind the data storage requirements. The final resolution was $0.634 /$ pixel which is sufficient to sample the PSF appropriately. The field of view provided by the chip was $5 ! 2 \times 5 ! 2$. Broad band Johnson's $B$ and $V$ and Kron-Cousin's $R$ and $I$ filters were used. About 3-4 exposures were taken in each of the photometric bands. The galaxy Mrk 87 was observed during two runs as the signal in the $B$ band was found to be inadequate after the first observing run. Twilight flats were taken and median filtered to construct the master flats. The data was reduced using $\mathrm{IRAF}^{1}$ on the IBM-6000 RISC system at PRL, Ahmedabad. Standard procedures of bias subtraction, flat-fielding and cosmic ray removal were done on the raw images. For the photometric calibrations of the $B V R I$ images, stars from Landolt (1992) were observed. The photometry of the standard stars was carried out using the DAOPHOT task in IRAF. Twenty standard stars from Landolt having $8{ }^{\mathrm{m}} 0<V<15^{\mathrm{m}} 0$ and $-0.20<B-V<+1.00$ were observed. Instrumental magnitudes were converted to standard magnitudes using the following equations obtained after a least squares fitting.

$(B-V)=(b-v)-0.48$

$(V-R)=1.05(v-r)-0.18+0.05(B-V)$

$(R-I)=0.94(r-i)+0.36+0.04(V-R)$

where lower cases denote instrumental values and upper cases correspond to the standard values. All the magnitudes and colours presented in this paper are in the $B$ and $V$ Johnson's and $R$ and $I$ Kron Cousin's system which is similar to the one used by Landolt to obtain the magnitudes and colours for the standard stars in his catalogue.

\footnotetext{
${ }^{1}$ IRAF is distributed by National Optical Astronomy Observatories, which is operated by the Association of Universities Inc. (AURA) under cooperative agreement with the National Science Foundation, U.S.A.
} 
Corrections for airmass were applied using the coefficients obtained from the transformation equations. The typical errors in the transformed magnitudes are 0.03 in $B, 0.03$ in $V, 0.02$ in $R$ and 0.04 in $I$. The galaxy images were shifted and co-added to improve the signal-to-noise ratio. The sky background was computed from the mode of the histogram of the image. The field of view was large enough compared to the program galaxies so that the histograms were sky dominated. The galaxy images were sky subtracted and subsequently scaled for 1 second exposure.

\section{Photometric accuracy}

In our final images, the noise per pixel has a standard deviation that corresponds to 24.5, 23.9, 23.8 and $23.0 \mathrm{mag} /{ }^{\prime \prime 2}$ in $B, V, R$ and $I$ respectively. We used published aperture photometry data compiled by Prugniel \& Heraudeau (1998) to test the accuracy of our values. Synthetic aperture photometry was carried out on the galaxies for which aperture photometry data exists in the literature and the results of the comparison are presented in Table 2. Our values agree very well with the values derived by other workers for most of the galaxies confirming the accuracy of our photometry. Our values for Mrk 14 and Mrk 87 are brighter as compared to the values obtained by Huchra. In case of Mrk 87, the difference is nearly the same within the limits of the errors and is most probably a result of an error in the determination of the zero-point for this observation. Mrk 213, Mrk 781 and Mrk 1379 were observed on the same night as Mrk 14. If the discrepancy in our values and those of Huchra would have been the result of an error in the zero-point determined from the transformations, the same systematic error would also have appeared for the other galaxies observed on the same night. However, no such effect is seen. Secondly, our values are brighter than those quoted in the literature which rules out inadequate exposure time as a cause of the discrepancy. Therefore, the difference appears to be genuine. One probable explanation for this could be a variability in the output luminosity of this source. Optical variability may be expected in young starbursts due to the appearance of supernovae (Terlevich 1992). In CCD photometry, the center of the galaxy can be determined with an accuracy of about one-tenth of an arcsec, while it is not possible to do so in aperture photometry. Small centering errors can also give rise to a discrepancy between the magnitudes derived from CCD and from aperture photometry, especially for small apertures.

\section{Surface photometry}

A direct inspection of the broad band images and the contour maps shows that many of these galaxies exhibit morphological peculiarities. To conduct a spatially resolved
Table 2. Comparison with aperture photometry from the literature

\begin{tabular}{lclllll}
\hline Galaxy & aperture & \multicolumn{2}{l}{ our values } & \multicolumn{2}{l}{ literature } & reference \\
Mrk & $\prime \prime$ & $V$ & $B-V$ & $V$ & $B-V$ & \\
\hline 14 & 15 & 14.67 & 0.42 & 14.81 & 0.42 & H \\
& 29 & 14.27 & 0.50 & 14.53 & 0.42 & $\mathrm{H}$ \\
87 & 15 & 14.42 & 0.80 & 14.53 & 0.86 & $\mathrm{H}$ \\
& 29 & 13.81 & 0.84 & 14.00 & 0.93 & $\mathrm{H}$ \\
& 57 & 13.16 & 0.81 & 13.35 & 0.90 & $\mathrm{H}$ \\
213 & 57 & 12.83 & 0.58 & 12.80 & 0.62 & $\mathrm{H}$ \\
363 & 24 & 14.02 & 0.53 & 14.03 & 0.57 & $\mathrm{H}$ \\
& 38 & 13.86 & 0.54 & 13.87 & 0.57 & $\mathrm{H}$ \\
449 & 81 & 12.97 & 0.66 & 12.99 & 0.66 & $\mathrm{H}$ \\
743 & 23 & 13.82 & 0.34 & 13.95 & 0.34 & M \\
1308 & 17 & 14.11 & 0.40 & 14.19 & 0.40 & HM \\
& 24 & 13.85 & 0.46 & 13.86 & 0.51 & HM \\
1379 & 15.4 & 13.6 & 0.61 & 13.67 & 0.58 & GHJ \\
& 26.8 & 13.21 & 0.63 & 13.44 & 0.65 & ARK \\
& 30 & 13.13 & 0.63 & 13.16 & 0.61 & GRI \\
& 37 & 13.01 & 0.64 & 13.02 & 0.63 & GRI \\
& 39.6 & 12.97 & 0.64 & 13.03 & 0.62 & GHJ \\
& 67.3 & 12.62 & 0.63 & 12.68 & 0.61 & GHJ \\
\hline
\end{tabular}

H: Huchra (1977); M: Mc Clure \& van den Bergh (1968); HM: Humay \& Maza (1987); GHJ: Griersmith et al. (1982); ARK: Arkhipova (1982); GRI: Griersmith (1980).

study of the stellar populations and the distribution of dust, we constructed $(B-V),(V-R)$ and $(B-I)$ colour maps. The colour maps were constructed after matching the PSFs in the two bands to avoid artefacts. This was achieved by degrading the better of the PSFs in the two bands by using a Gaussian smoothing filter. The colour maps thus obtained were examined to identify features like star forming regions, dust lanes, etc. and study their photometric properties and locations with respect to the underlying galaxy. See Figs. 1a-j. Grey scales have been chosen to maximize the contrast over the range of colour indices.

The surface brightness distribution and the variation of the position angle and ellipticity of the isophotes of each galaxy were obtained using the ISOPHOTE package within STSDAS ${ }^{2}$. Ellipses were fit to the galaxy images in all the four pass bands after masking out any foreground stars. The ellipse fitting was done using the algorithm proposed by Jedrejewski (1987). The deviation of an isophote from a perfect elliptical shape can be estimated by expanding the difference in intensity between the isophote and the corresponding fitted ellipse as a Fourier series in the eccentricity anomaly along the isophote. A negative coefficient B4 of the $\cos (4 \phi)$ term indicates boxiness while a positive coefficient indicates the presence of a disk. Though this technique was initially used in the analysis of elliptical galaxies, in recent years, it has been successfully used in identifying structures like bars, isophotal twists, dust lanes, etc. in disk galaxies also (Wozniak et al. 1995;

\footnotetext{
${ }^{2}$ The Space Telescope Science Data Analysis System STSDAS is distributed by the Space Telescope Science
} Institute. 


\section{MRK 14}
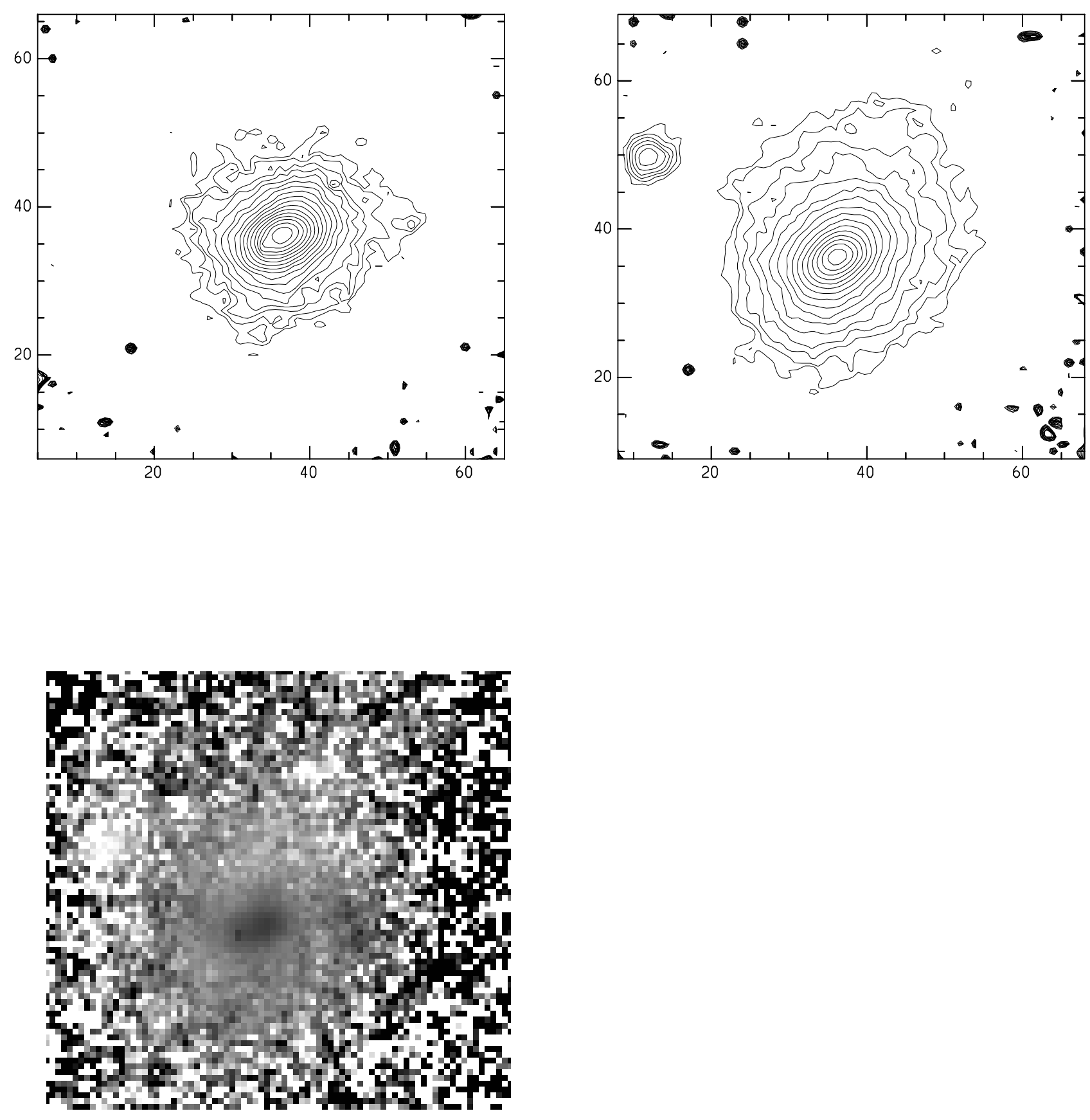

Fig. 1. a) Clockwise from top left, the $B$ and $I$ band isophotes, the residual image and the $(B-I)$ image. The contours are plotted between $19-23 \mathrm{mag} /{ }^{\prime \prime 2}$ at intervals of $0.5 \mathrm{mag} /{ }^{\prime \prime 2}$ for the $B$ band and between $17-22 \mathrm{mag} /{ }^{\prime \prime 2}$ with the same interval for the $I$ band. The same order is followed in the figures a) to j). For $(B-I)$ images, dark is blue and white is red. For Mrk 87 , the $B$ and $I$ images are presented instead of the isophotes to depict the inner ring clearly. North is at the the top and east is to the left. Residual images in the $R$ band are given only for those galaxies which show some fine structure 


\section{MRK 87}
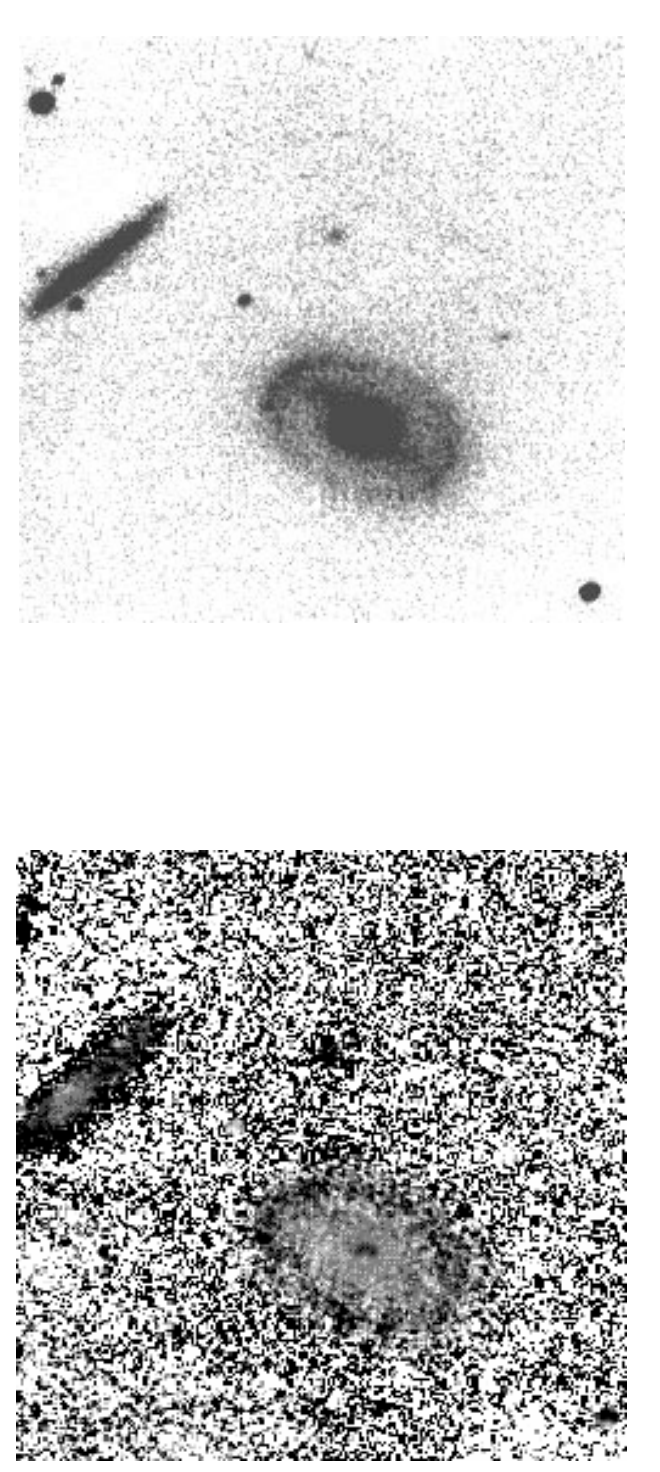

Fig. 1. b) continued

Wozniak \& Pierce 1991). Analysis of a sample of MBG starbursts has been carried out by Barth et al. (1995) using ellipse fitting techniques. Non-circular motions of the gas increase the rate of collisions, as well as the radial transport of gas to the galactic center and are seen as isophotal twists. Scoville \& Hersh (1979) suggest that this mechanism could be responsible for enhancing the gas density in the nucleus and triggering star formation there. Generally, all the parameters of the ellipses, viz ellipticity, position angle and center, were allowed to vary. However, in Mrk 743, which shows a double nucleus, the center was kept fixed at a value derived from the outermost isophotes. $-10^{\prime \prime}$

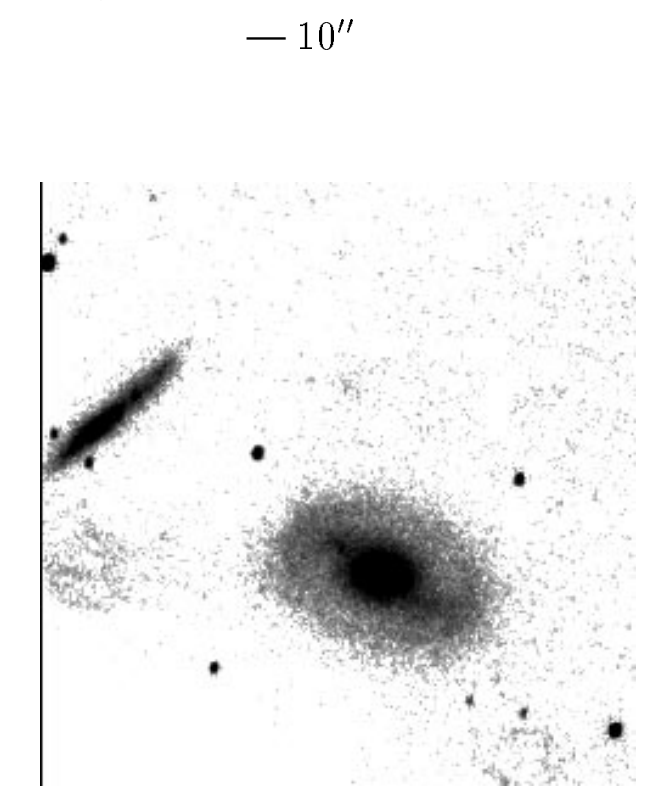

Ellipses were fit right up to the central pixel in all cases except in Mrk 743 due to its double nucleus and in Mrk 363 which does not show a well defined nucleus in the $B$ band. For these galaxies, the minimum radius of fitting was decided on the basis of the extent of the central structure. The results of the ellipse fitting viz. the radial variation of the surface brightness, the ellipticity (e) and the position angle (PA) are presented in Figs. 2a-i. The variation in colour is presented in Figs. 3a-j. From the ellipticity of the outermost fitted isophotes, we derive the angle of projection of the galaxy on the plane of the sky using the equation given by Tully (1988). 


\section{MRK 213}
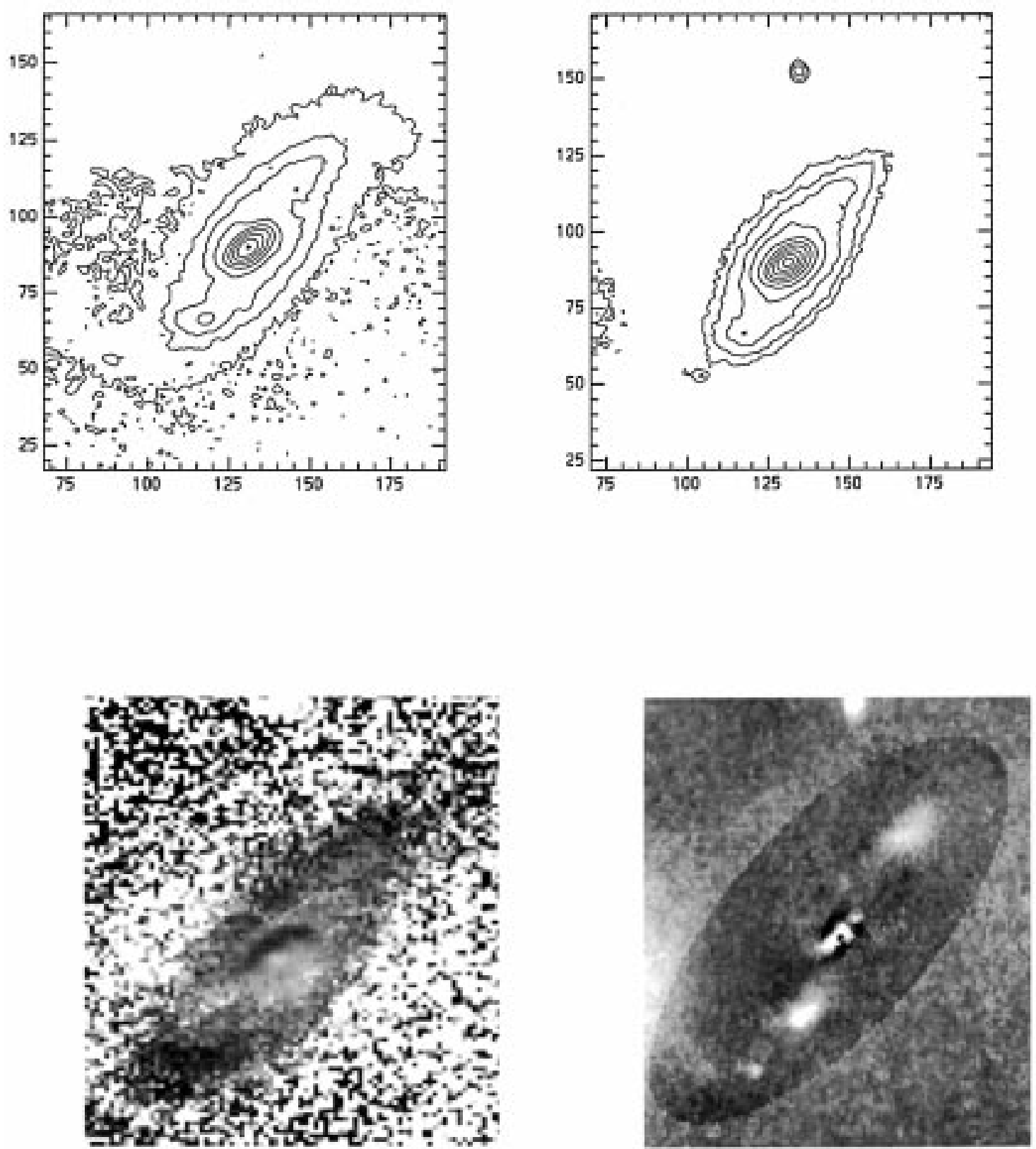

Fig. 1. c) continued

$$
\begin{aligned}
& i=\cos ^{-1}\left\{\frac{(b / a)^{2}-0.2^{2}}{1-0.2^{2}}\right\}^{1 / 2}+3^{\circ} \\
& e=1-\frac{b}{a} .
\end{aligned}
$$

The results are tabulated in Table 1 . The total light enclosed within the isophote at 24, 23.5, 23.5 and 22.5 $\mathrm{mag} /{ }^{\prime \prime 2}$ in the $B, V, R$ and $I$ images respectively, was

used to determine $m_{B}, m_{V}, m_{R}$ and $m_{I}$ by summing over the light in the fitted elliptical annuli. The magnitudes derived in this manner and colour indices for each galaxy are presented in Table 3. along with the spatial distribution of the starburst.

In order to identify non axisymmetric structure and other small scale features, unsharp masked images were constructed for each galaxy. For this, we produced models 


\section{MRK 363}
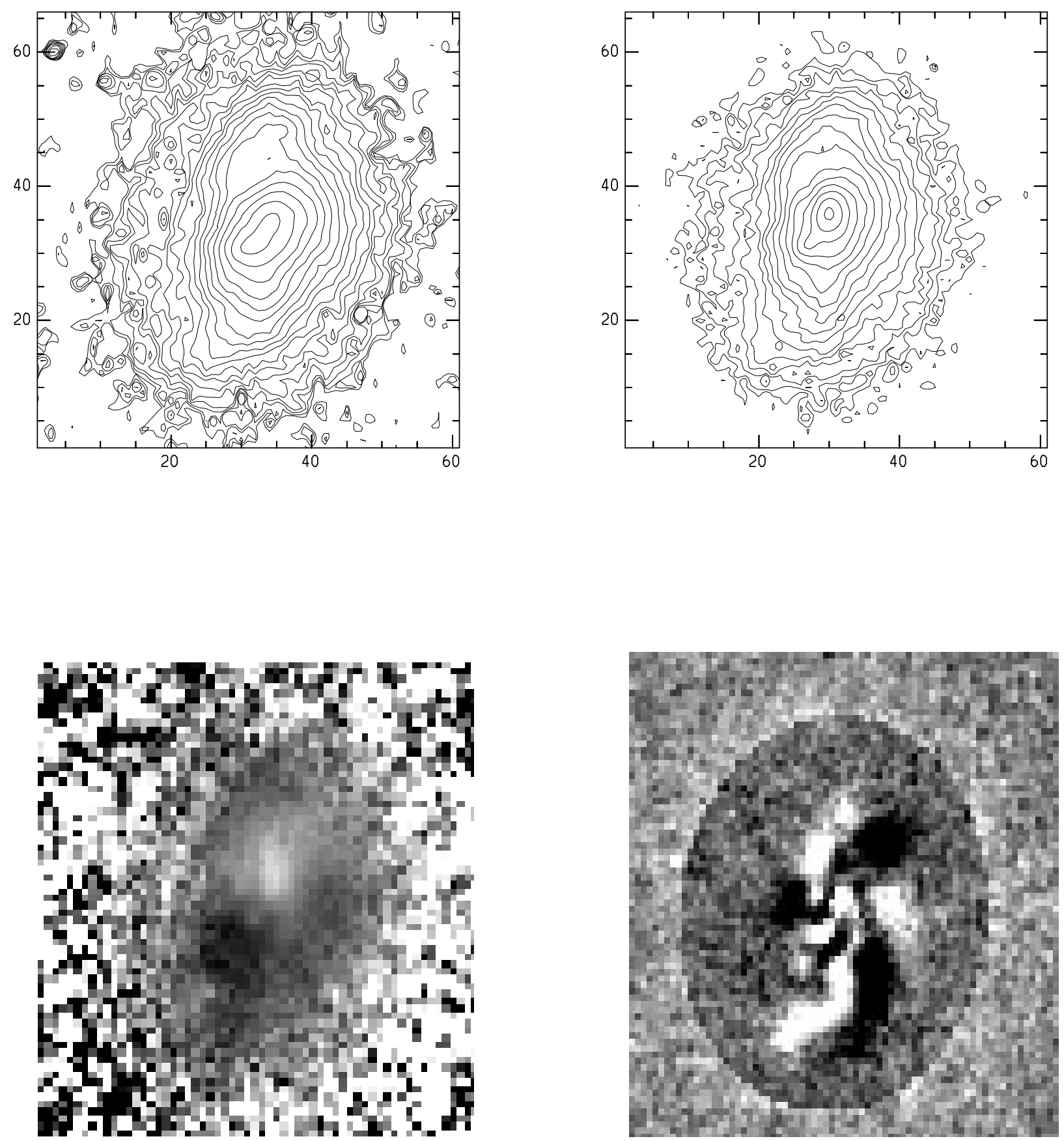

Fig. 1. d) continued

for the smooth light distribution of the galaxies in each band pass using the parameters derived from the isophotal analysis. This smoothed image was subtracted from the original image, to produce a residual image with enhanced features not apparent in the direct images. Such fine structure provides a wealth of information about the processes at play in the galaxy. We detect complex fine structure most prominently in the galaxies Mrk 1002, Mrk 363 and Mrk 213. For a detailed discussion refer to Sect. 4. Schweizer \& Seitzer $(1988,1992)$ quantify such fine structure in E and S0 galaxies that are believed to be induced by mergers. Simulations by Hernquist (1992, 1993), also predict features like boxy isophotes and $\mathrm{X}$-shaped fine structure for merger products. 


\section{MRK 449}
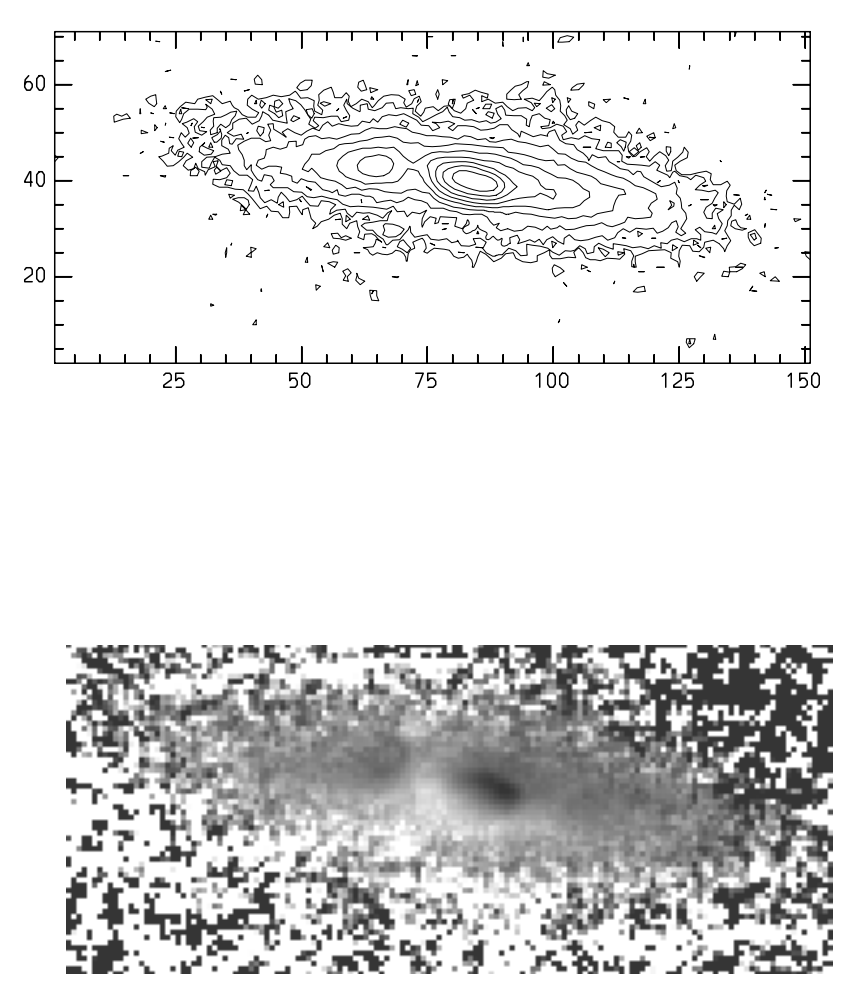

Fig. 1. e) continued

\section{Notes on individual galaxies}

1. Mrk 14: This galaxy has been classified as likely to be an S0 by Huchra (1977), while it forms a part of the sample of distant irregulars in a study conducted by Hunter \& Gallagher (1986). The contours in the outer regions look disturbed. See Figs. 1a and 2a. The ellipticity profiles show a complex structure. Ellipticity is highest $(\approx 0.3)$ in the central region in all the filters. It drops to a value of 0.1 at $9^{\prime \prime}$. Beyond $9^{\prime \prime}$ the ellipticity rises again, showing a number of peaks before reaching a value of 0.2 in the outermost region. The isophotes show a continuous twist $\left(40^{\circ}\right)$ from the central to the outer regions. The contours in the inner $8^{\prime \prime}$ show a boxy nature while beyond $10^{\prime \prime}$ they become pointy as is seen from the negative and the positive values respectively of the coefficient B4 of the $\cos (4 \phi)$ term depicted in Fig. 4 . Keel \& van Soest (1992) find no candidate companions near this galaxy. The only significant feature in the colour maps is the blue nucleus (Fig. 1a). The $(B-I)$ colour map does not show any features like dust lanes which can be responsible for the strong isophotal twist. The variation of the colour indices, $(V-I),(B-R)$ and $(B-V)$ with distance from the center are shown in Fig. 3. On the whole, the colours get redder outwards. A sharp change is seen in the inner $8^{\prime \prime} .(B-V)$ changes steeply from 0.15 to 0.75 in this region. Beyond this, the change is more gradual. The residual image constructed with the model light distribution subtracted from the direct image fails to reveal any other structure besides a bright nuclear region. A strong isophotal twist and boxiness of the isophotes are indicative of possible interaction in the past. Similar inferences have been drawn by Nieto \& Bender (1989) and Bender \& Möllenhoff (1987) for other early-type galaxies. This is the most likely cause for triggering the central starburst in Mrk 14. Besides interactions in the past, an alternative scenario has been suggested by the referee which is also capable of explaining the observed features like the peak in ellipticity, the isophotal twisting and boxiness. He suggests that the above features could also arise from a triaxial bulge remnant of a bar destroyed by the gas accumulation in the nucleus. For such a 

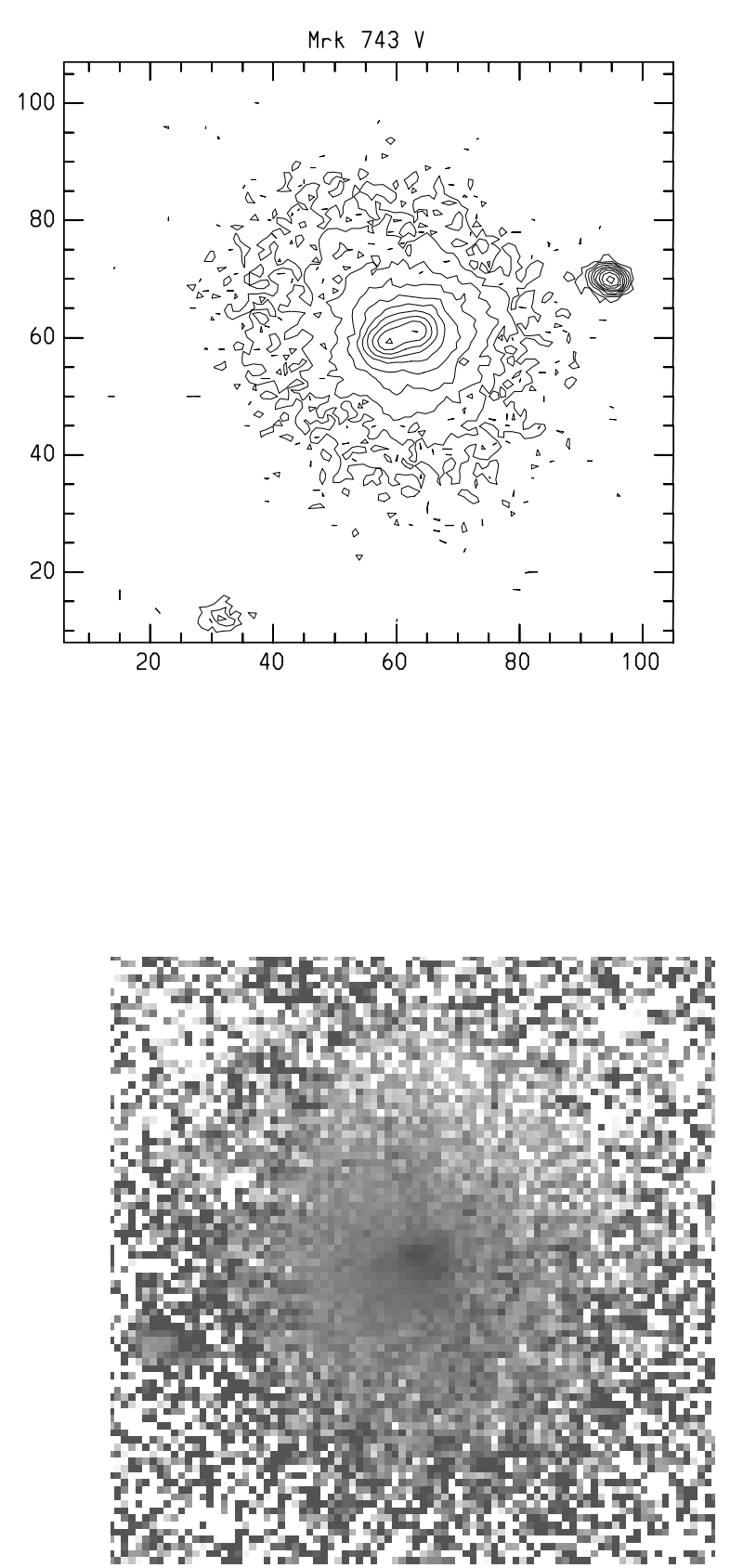

Fig. 1. f) continued

scenario, the colour gradients can be explained by the star formation that occurred during the gas inflow, the twisting of isophotes could be due to the triaxial bulge and the boxiness as well as the ellipticity peak could be a signature of the now disappeared bar. Hence, the observed features in Mrk 14 could be expected to arise either as a result of a past interaction or the formation of a triaxial bulge from a bar due to mass inflow towards the center.

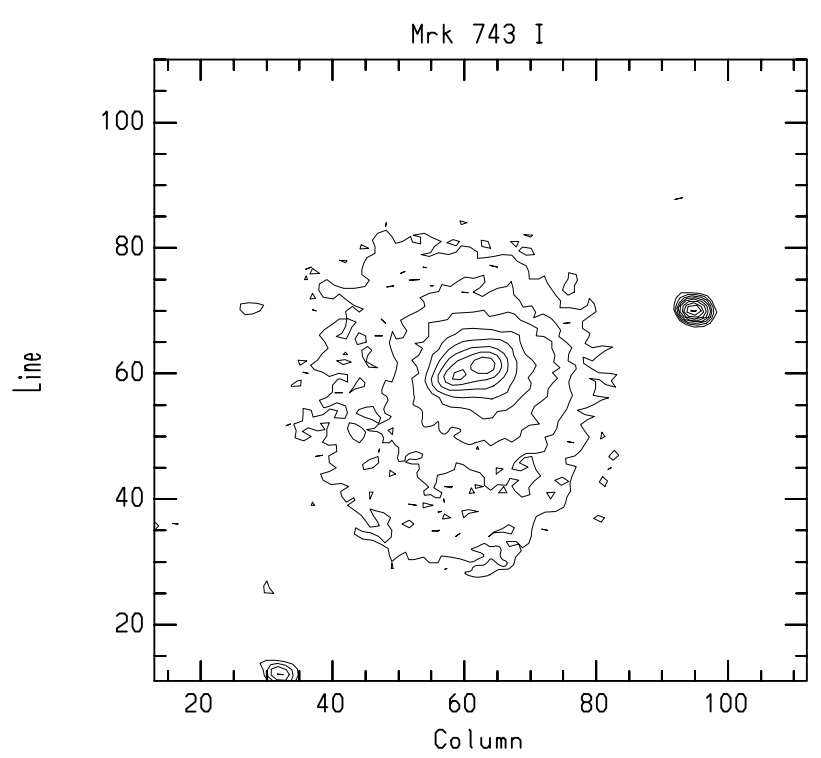

2. Mrk 87: This is an example of a ringed galaxy. It shows a bright nucleus and a bar surrounded by an inner ring. The nucleus, the bar and the inner ring appear prominently in the $B$ band image. Towards longer wavelengths, the light distribution becomes smoother (Fig. 1b). A companion is situated to the NE of Mrk 87. The central regions seem to be disturbed due to the presence of dust as indicated by the filter dependent behaviour of the ellipticity and the position angle in the inner $5^{\prime \prime}$ in Fig. 2b. Beyond 
MRK 781 $10^{\prime \prime}$
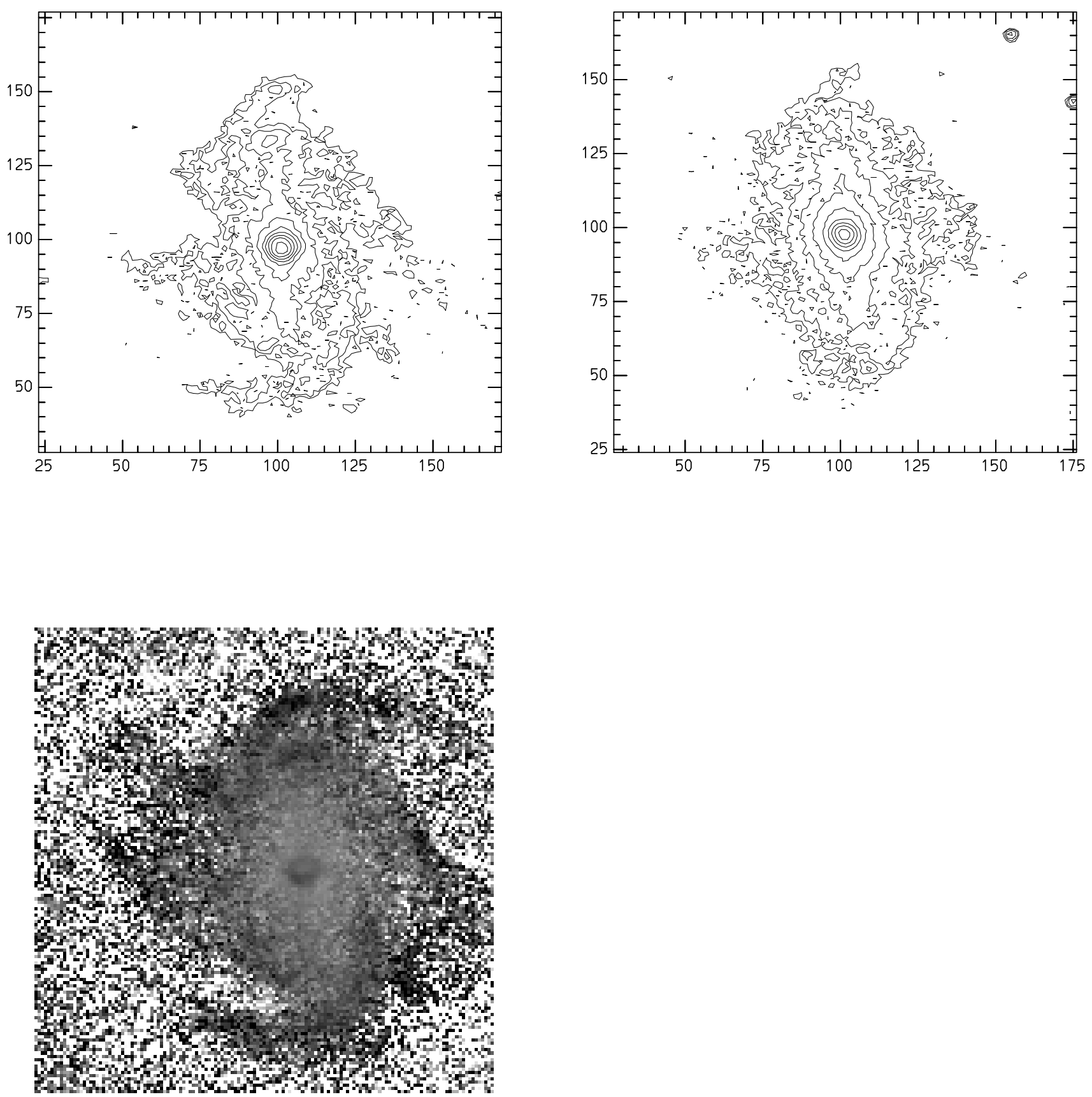

Fig. 1. g) continued

5 ", the behaviour is similar in all filters. The ellipticity profiles show a slight discontinuity at $8^{\prime \prime}$. Between $5^{\prime \prime}$ and $10^{\prime \prime}$, the position angle in nearly constant at $75^{\circ}$ and then slowly changes by $15^{\circ}$ before reaching the final value of $60^{\circ}$. The presence of the blue ring is also clearly seen in Fig. 3 at $24^{\prime \prime}$. Rings of stars and gas are often seen in barred spiral galaxies. They are believed to be formed by gas accumulation at the bar's Lindblad resonances. They are blue in colour and are the sites of enhanced star for- mation (Buta 1986). Arsenault (1989) has shown that the bar and ring features occur with a higher frequency in starburst galaxies as compared to normal galaxies.

3. Mrk 213: This is a barred spiral galaxy. A faint arm is seen emerging from the south-eastern end and curving around towards the north-western side where it is attached to an almost stellar condensation. The contour plots (Fig. 1c) clearly show that the contours in the inner regions have a position angle different from that of the outer 

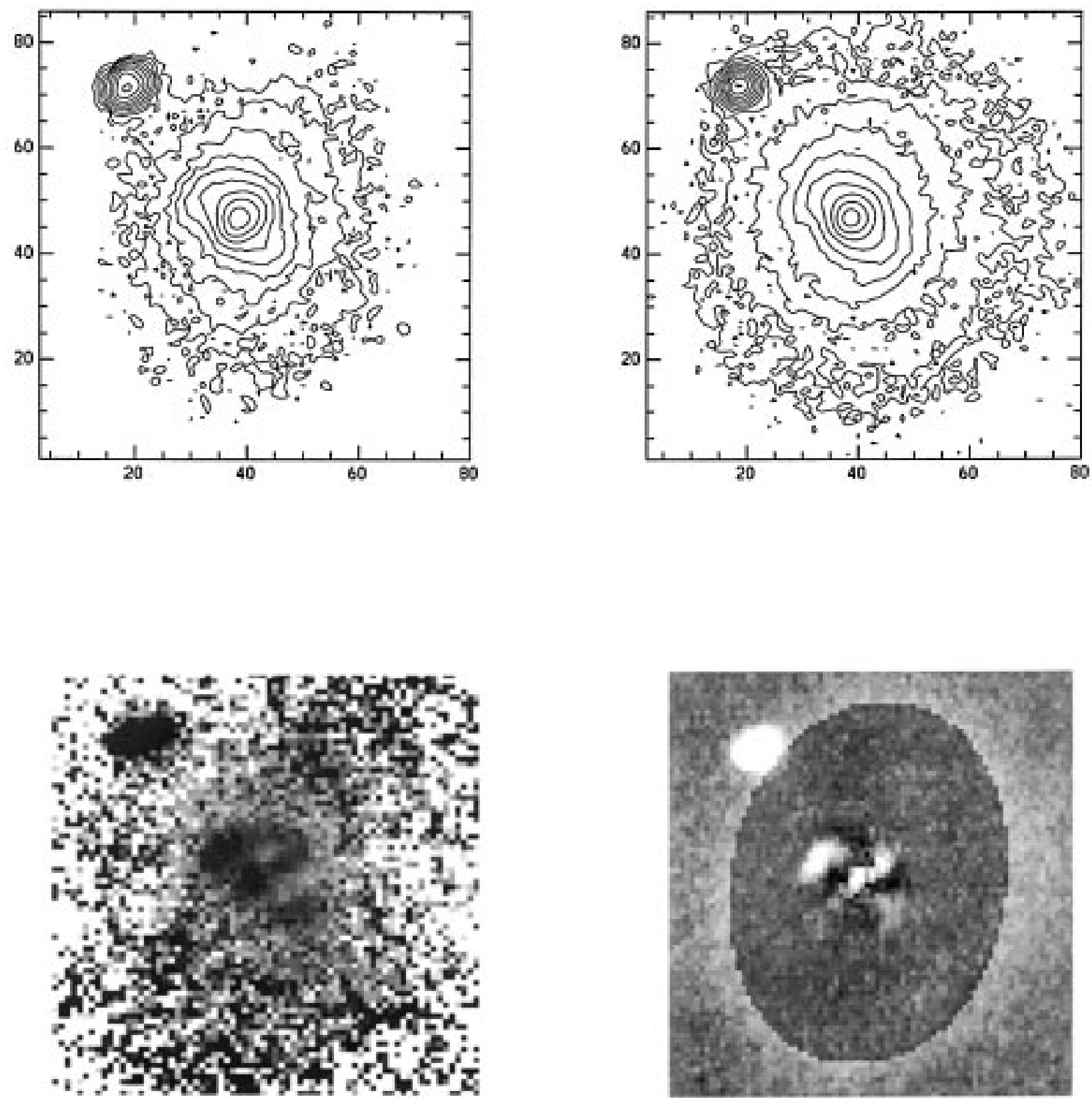

Fig. 1. h) continued

region. The contours in the central region appear elliptical. A very bright, saturated star is present at $58^{\prime \prime}$ from the center of the galaxy. This star was first masked out before starting the ellipse fitting procedure. The ellipticity profiles show a peak at $4^{\prime \prime}$. Beyond this, the ellipticity falls and then rises again to the disk ellipticity of about 0.5 . The position angle curves show a nearly constant value in the inner $8^{\prime \prime}$. We attribute these features to the presence of a nuclear bar in the central region. Such nuclear bars have been detected by Jungwiert et al. (1997) and references therein and Wozniak et al. (1995) in a number of disk galaxies. These bars are believed to be an efficient mechanism for driving gas into the nuclear region and fueling the starburst. The position angle changes by $25^{\circ}$ between $8^{\prime \prime}$ and $10^{\prime \prime}$ (Fig. 2c). A dust lane starts from the nucleus, curves around it before proceeding towards the NW 


\section{MRK 1308}
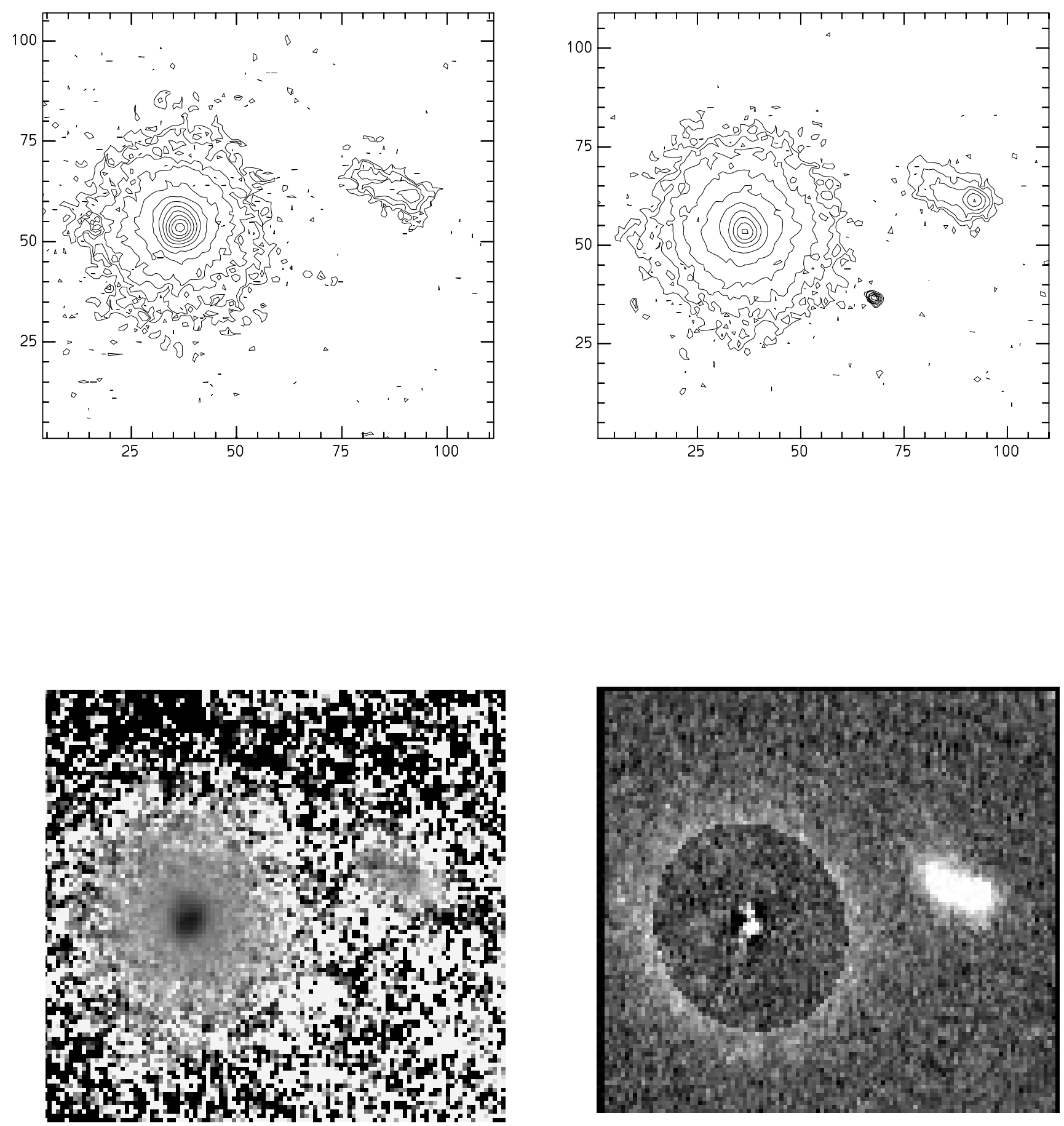

Fig. 1. i) continued 

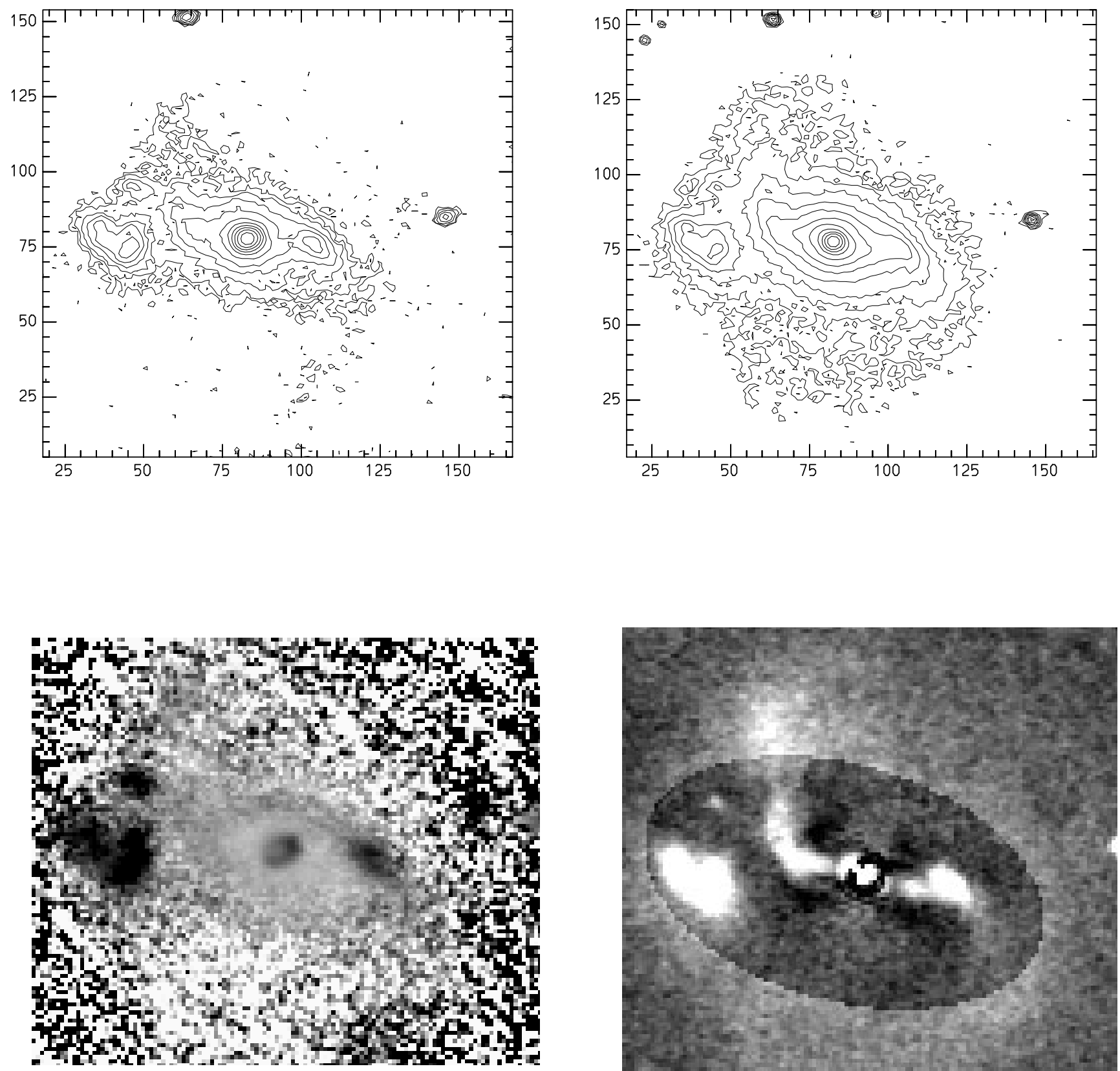

Fig. 1. j) continued

direction. The residual maps also show the signature of the bar in the form of a linear structure in the central region (see Fig. 1c).

4. Mrk 363: This peculiar Sc type galaxy has been classified by Geller \& Huchra (1983) as belonging to a group made up of seven galaxies, based on their proximity in space and their radial velocities. A neutral hydrogen mapping by van Moorsel (1988) shows a central concentration of HI. Radio emission extended over the galaxy has been observed by Wrobel \& Heeschen (1988).
An inspection of the contours in the $B$ and the $I$ band (Fig. 1d) reveals that the morphologies are quite different in the two spectral windows. The blue continuum has an extended structure in the central region with no well defined nucleus while the $I$ band image shows a well defined nucleus in the central region. The contours appear highly disturbed and asymmetric in both the bands, though they are more so in the $B$ band. The contours in the $B$ band are stretched out in the north more than those in the $I$ band suggesting the presence of dust in the region at $\approx 9^{\prime \prime}$ from 

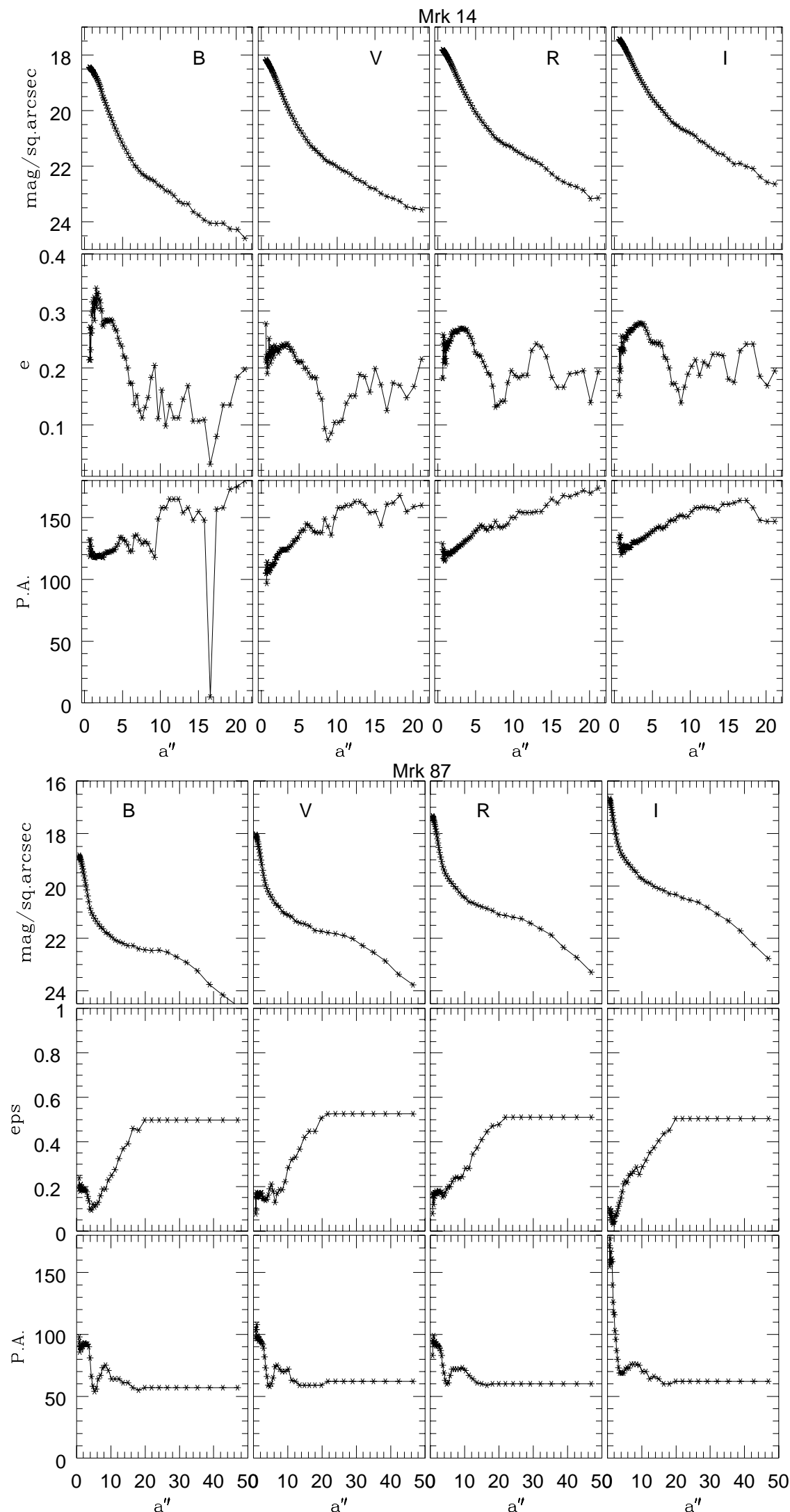

Fig. 2. a,b) Radial variation of surface brightness, ellipticity (e) and position angle (PA) in $B, V, R, I$ from left to right 

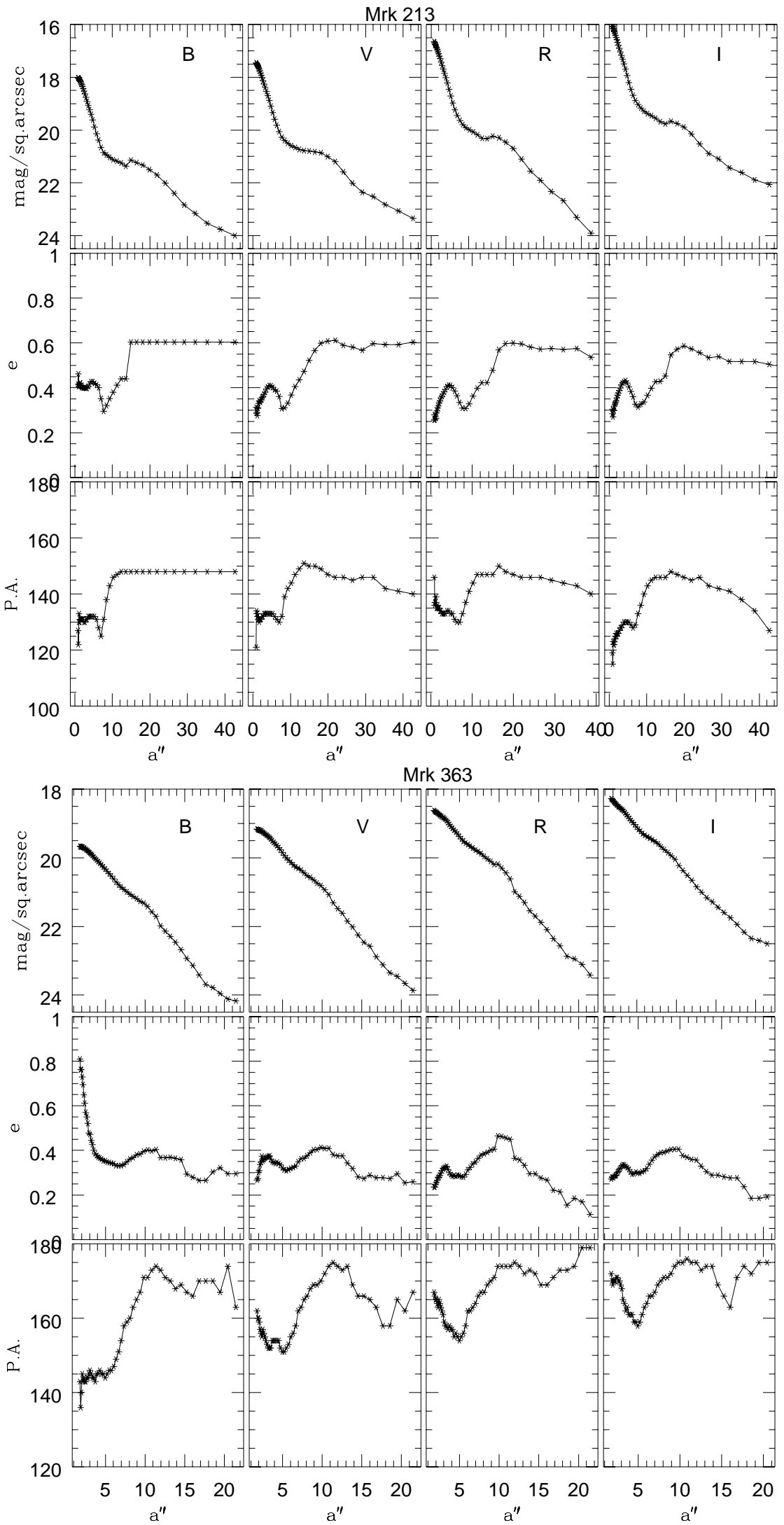

Fig. 2. c,d) continued 

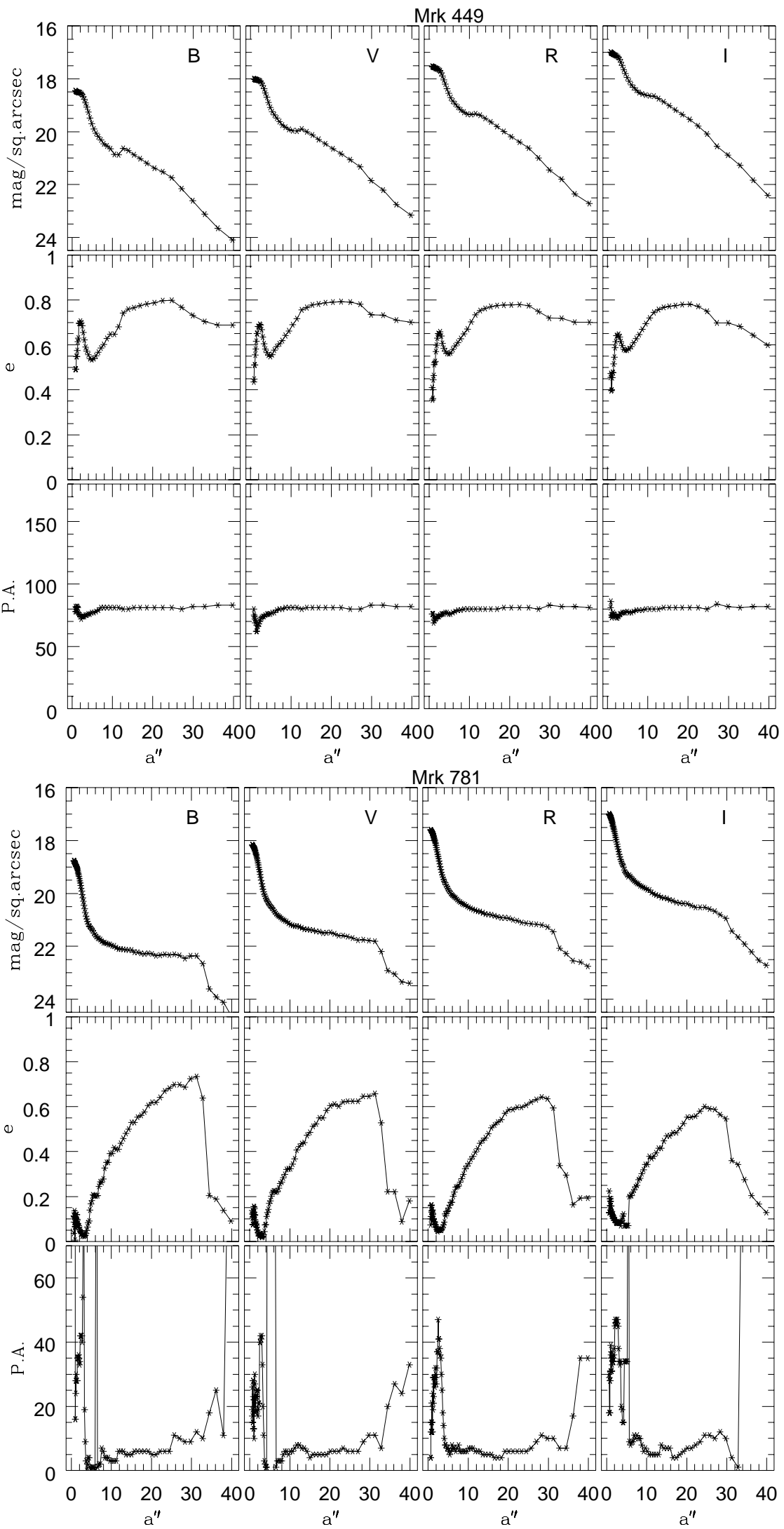

Fig. 2. e,f) continued 

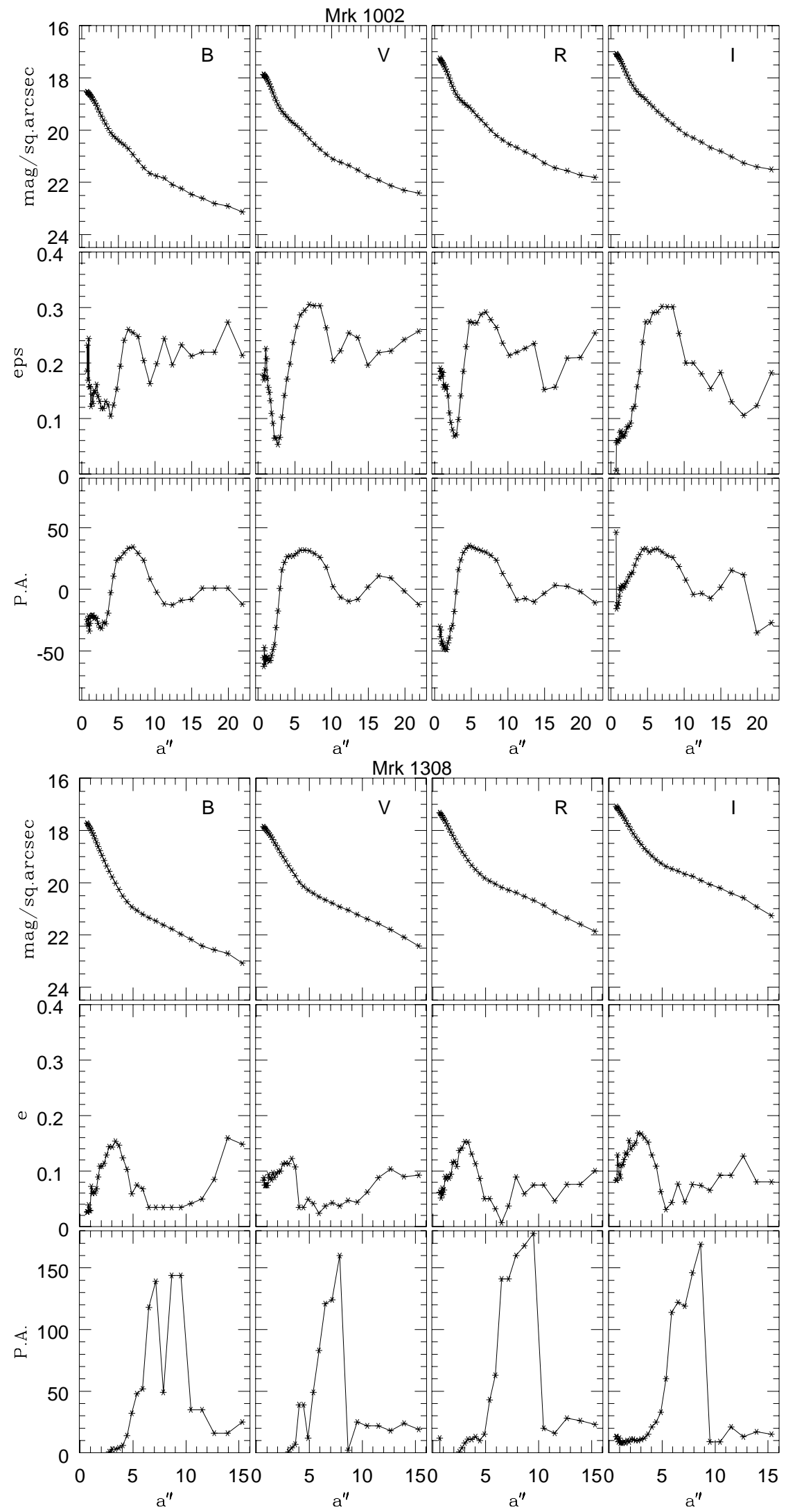

Fig. 2. g,h) continued 


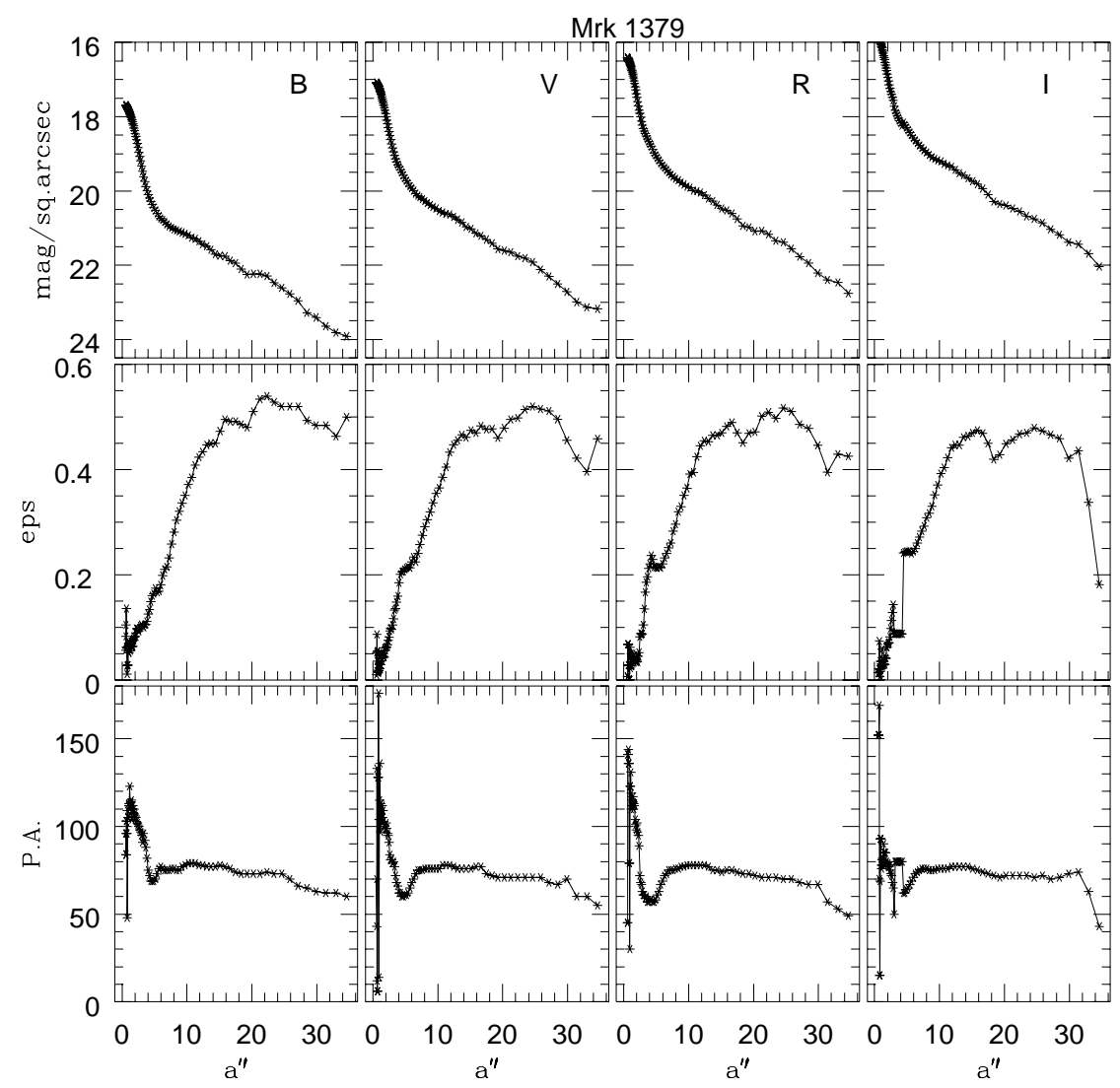

Fig. 2. i) continued

Table 3. Total magnitudes and colour indices uncorrected for galactic absorption and inclination effects. The last column describes the locations of the starburst, where nuc: nuclear, comp: companion galaxy

\begin{tabular}{|c|c|c|c|c|c|c|c|c|c|c|c|}
\hline \multirow[t]{2}{*}{ Mrk } & \multicolumn{2}{|c|}{$\overline{B_{\mathrm{T}}}$} & \multicolumn{2}{|c|}{$\overline{V_{\mathrm{T}}}$} & \multirow[t]{2}{*}{$R_{\mathrm{T}}$} & \multirow[t]{2}{*}{$\overline{I_{\mathrm{T}}}$} & \multirow[t]{2}{*}{$m_{B}$} & \multirow[t]{2}{*}{$m_{B}-m_{V}$} & \multirow[t]{2}{*}{$m_{V}-m_{R}$} & \multirow[t]{2}{*}{$m_{R}-m_{I}$} & \multirow[t]{2}{*}{ starburst } \\
\hline & this work & RC3 & this work & RC3 & & & & & & & \\
\hline$\overline{14}$ & 14.67 & 14.9 & 14.11 & 14.49 & 13.54 & 13.09 & 14.74 & 0.52 & 0.51 & 0.47 & nuc \\
\hline 87 & 13.87 & 13.8 & 12.77 & 12.85 & 12.04 & 11.49 & 14.12 & 0.84 & 0.67 & 0.71 & nuc+ring \\
\hline 213 & 13.18 & 13.10 & 12.68 & 12.50 & 12.06 & 11.44 & 13.39 & 0.60 & 0.54 & 0.59 & nuc+bar end \\
\hline 363 & 14.31 & 14.28 & 13.76 & 13.72 & 13.20 & 12.81 & 14.34 & 0.52 & 0.56 & 0.32 & global \\
\hline 449 & 13.45 & 13.50 & 12.87 & 12.85 & 12.39 & 11.69 & 13.69 & 0.66 & 0.53 & 0.63 & nuc \\
\hline 743 & 13.19 & - & 13.04 & - & 12.67 & 12.06 & 13.57 & 0.36 & 0.47 & 0.63 & one nucleus \\
\hline 781 & 13.07 & 13.19 & 12.42 & - & 11.92 & 11.44 & 13.40 & 0.64 & 0.53 & 0.59 & nuc+spiral arms \\
\hline 1308 & 14.04 & - & 13.45 & - & 12.99 & 12.39 & 14.24 & 0.50 & 0.47 & 0.52 & nuc \\
\hline 1379 & 13.29 & 13.00 & 12.59 & 12.34 & 12.04 & 11.31 & 13.43 & 0.63 & 0.58 & 0.77 & nuc+bar end+comp \\
\hline
\end{tabular}

the nuclear region. The $(B-I)$ map clearly shows a highly reddened region coinciding with this feature. This region has a mean $(B-V)$ of 0.7 while the nuclear region has a mean value of 0.3 . The $I$ band image shows a pointed structure starting from the nuclear region and extending up to $4^{\prime \prime}$ along the SE direction. There is another pointy structure starting at $6^{\prime \prime}$ and extending up to $12^{\prime \prime}$ along the southern direction. The ellipticity in the central and the outer regions is 0.2 as is seen from Fig. 2d. Close to the nucleus, the ellipticity profile shows a small kink. It rises to a value of 0.4 at $9.5^{\prime \prime}$ - the region where the contours start becoming pointy again. Subtracting the smoothed image from the direct images revealed complex fine struc- ture. A bright and stellar nucleus, with spiral arms lying along the north-south direction and extending right into it are seen in Fig. 1d. As this galaxy belongs to a group of seven galaxies, tidal interactions with the other galaxies of the group are a likely cause of the enhancement in star formation activity in this galaxy.

5. Mrk 449: This is the most inclined galaxy in our sample. We derive an inclination of $75^{\circ}$ for this galaxy. The contour maps show the presence of a highly reddened region lying to the east of the central nucleus. This feature gets weaker towards longer wavelengths. Correspondingly, the colour map also shows a reddened vertical band in this region (refer Fig. 1e). We interpret this as a dust lane. This 
Mrk 14
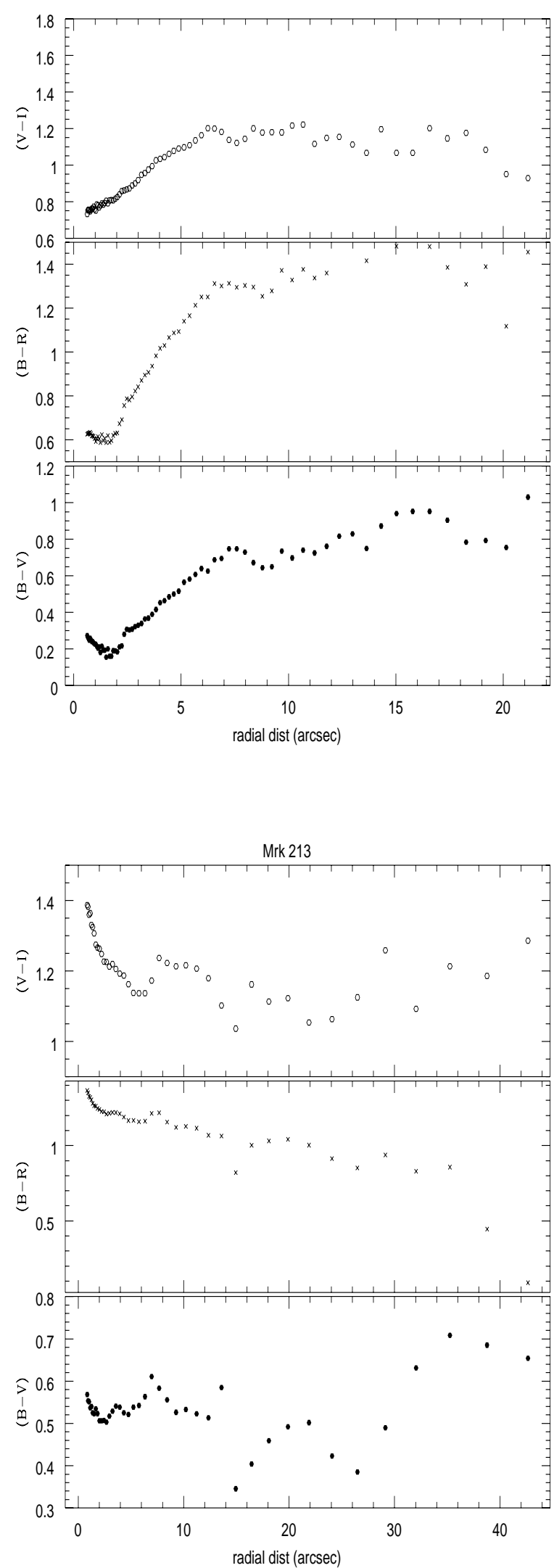

Fig. 3. Radial distribution in the colour indices for the galaxies
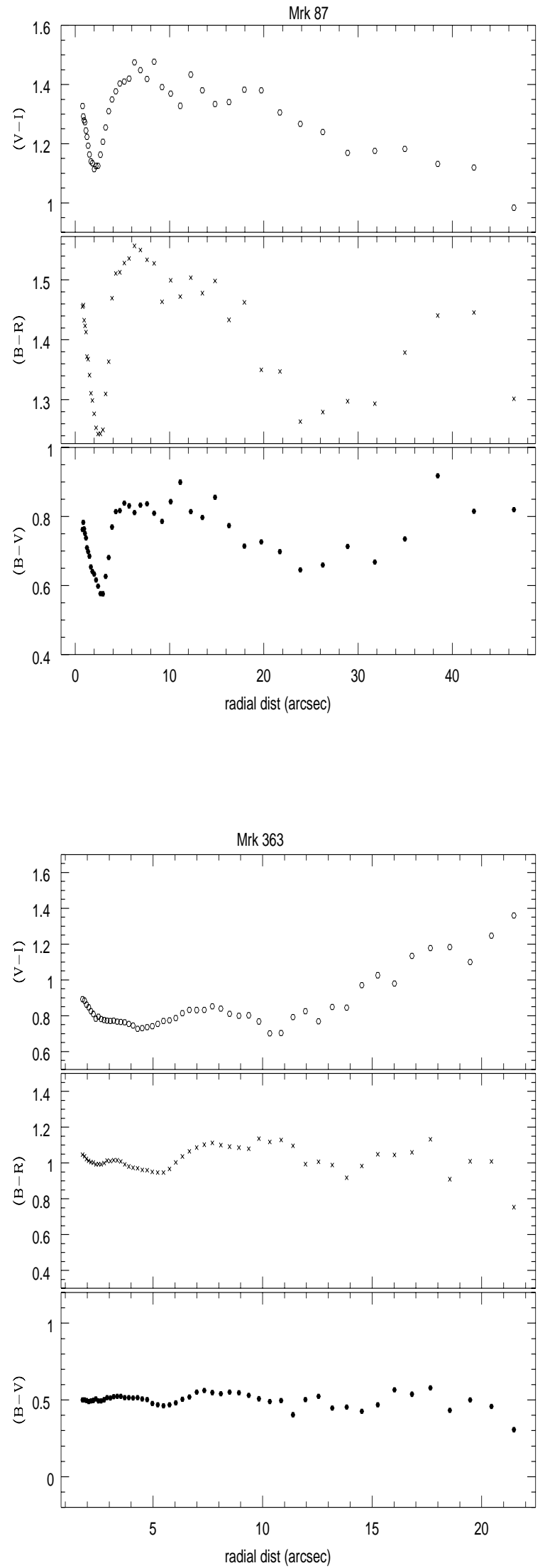

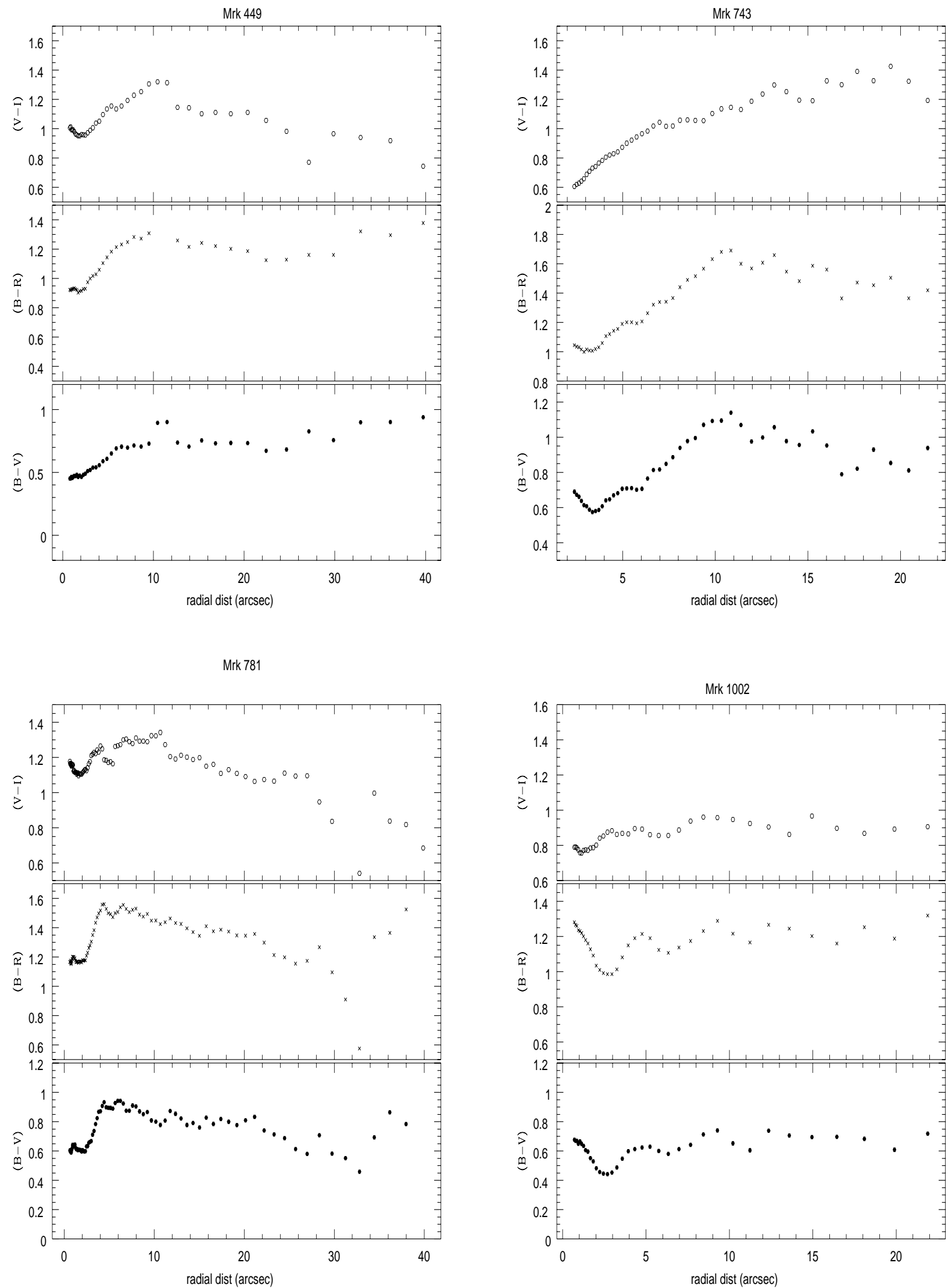

Fig. 3. continued 

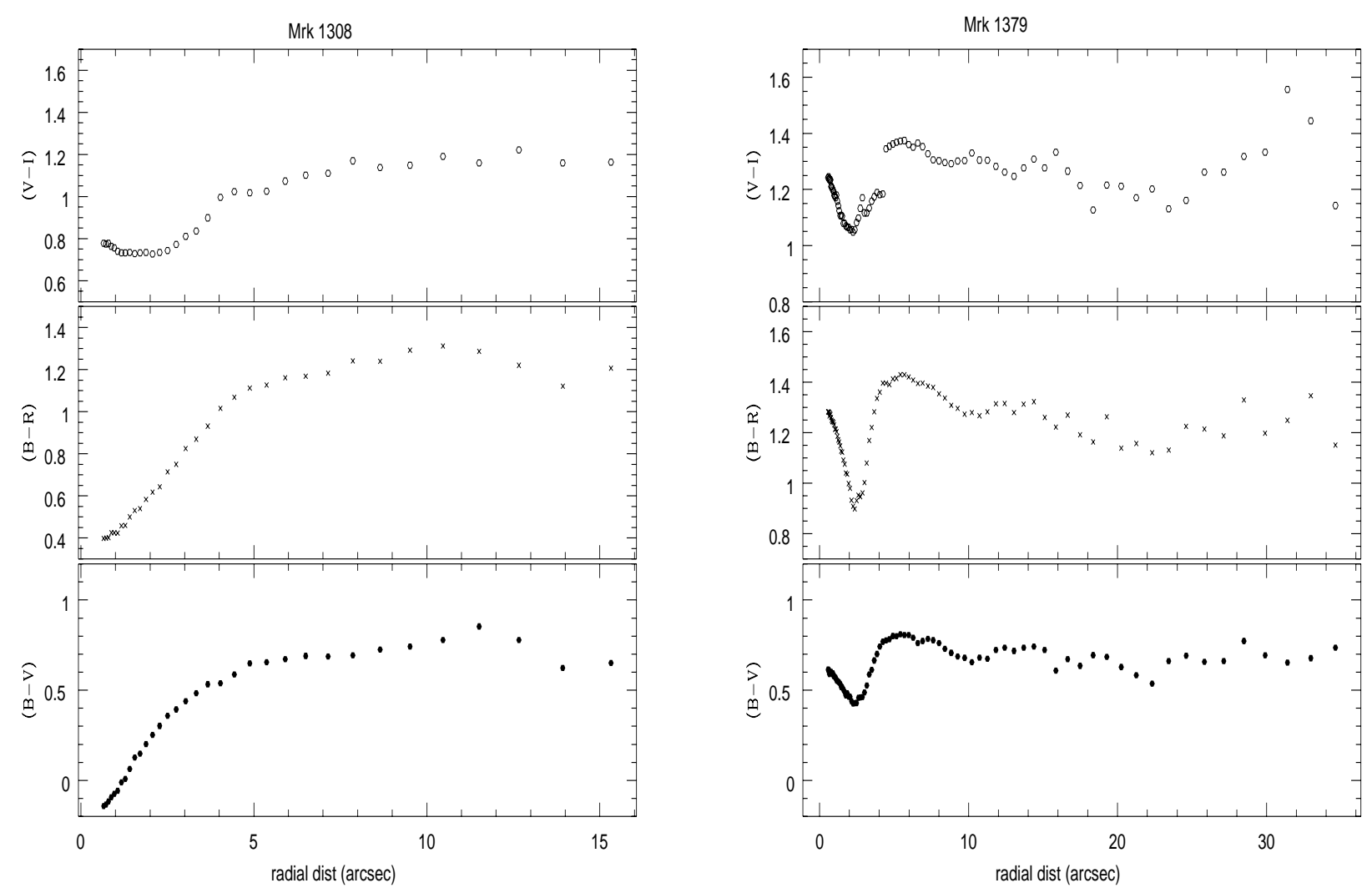

Fig. 3. continued

dust lane lies neither along the major axis of the galaxy, nor along its minor axis, but is at an intermediate angle. Hawarden et al. (1981) observed such "skew" dust lanes in a small fraction of early type galaxies. They suggest that the properties of the galaxies with such "skew" dust lanes are best attributed to the accretion of cool material at a fairly recent epoch. The signature of this is also seen in the surface brightness profiles as a dip at $10^{\prime \prime}$ most prominent in $B$ and weakest in $I$ (Fig. 2e), and as a reddened region in the colour plots in Fig. 3. A cut across the dust lane taken at a position angle $97^{\circ}$ is presented in Fig. 5, which clearly shows the extent and the reddening due to the dust lane in $(B-R) .(B-R)$ changes from 0.8 in the nuclear region to 1.5 at the location of the dust lane. The position angle remains constant throughout except for a small dip in the central region. The very high inclination of this system makes it difficult to interpret the results of the ellipse fitting uniquely. The residual image shows complex fine structure. Excess of luminosity is present in the nucleus as well as in the region to the east of the dust lane.

6. Mrk 743: is classified as a peculiar E0 galaxy in the Markarian catalog. It forms a part of the sample of galaxies with double nuclei studied by Mazzarella \& Boroson (1993). An inspection of the contour plots in the four bands reveals the presence of two nuclei surrounded by a common envelope. The envelope is asymmetric in the outer regions. Both the nuclei have comparable fluxes in the $V$ band. As we go towards longer wavelengths, the western nucleus starts getting brighter, while the eastern component becomes dominant at shorter wavelengths (Fig. 1f). Mrk 743 is one of the few HI sources among early type galaxies in which the HI distribution shows a central concentration, rather than the usual depression (Burstein et al. 1987). The HI distribution is in the form of a disk nearly as large as the galaxian diameter (van Driel \& van Woerden 1991). Wrobel \& Heeschen (1991) detect unresolved radio emission at $6 \mathrm{~cm}$ from this object. We fitted ellipses to this galaxy keeping the center coordinates fixed at a point between the two nuclei. This was maintained for all the filter bands. Ellipses were fit only in the common envelope region. The surface brightness profile is shown in Fig. 6. The colour plots in Fig. 3 show a sharp change in the inner $10^{\prime \prime}$. Since Mrk 743 shows such a peculiar morphology, one cannot comment on the nature of the underlying galaxy from the results of the ellipse fitting process. However, the presence of two nuclei with star formation enhanced in only one and the presence of an asymmetric outer envelope all indicate that this is a merger in progress.

7. Mrk 781: This is is a barred galaxy with a flocculent spiral structure. No conspicuous difference is seen in the structure between the $B$ and $I$ wave bands in Fig. 1g. We detect a blue ring surrounding the nuclear region in the colour images. Besides the ring and the blue spiral arms, the rest of the galaxy has $(B-V)$ between 


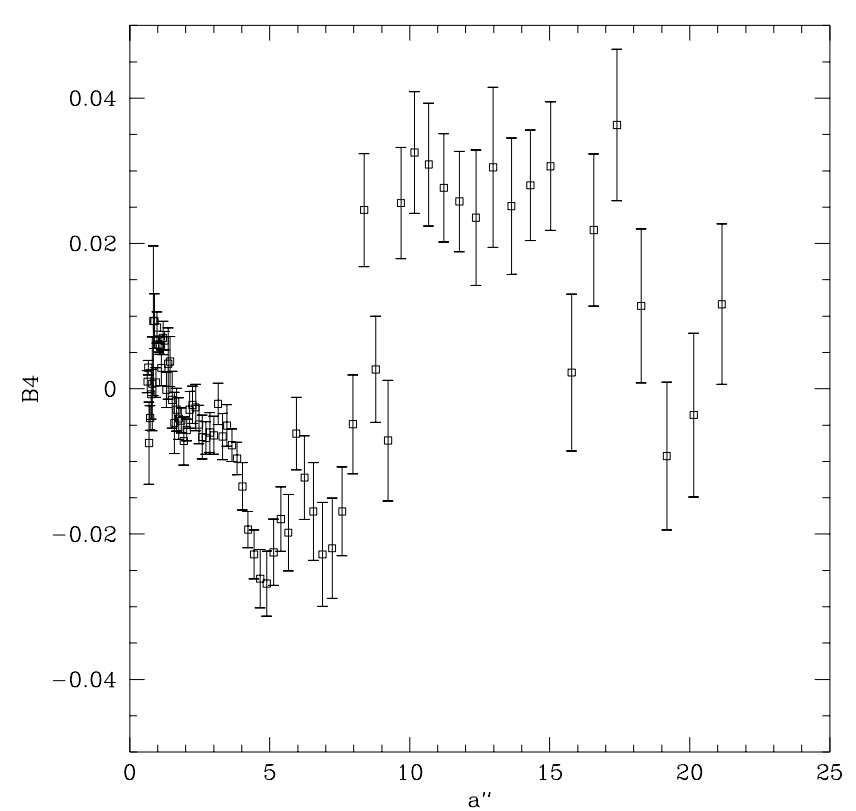

Fig. 4. The B4 coefficient as a function of the semi-major axis for Mrk 14

$0.8-0.9$. The extent of the bar is estimated from the peak in the ellipticity profiles in Fig. $2 \mathrm{~g}$ to be $30^{\prime \prime}$. However, there is a systematic shift in the peak ellipticity from $B$ to $I$ with the peak in $B$ having the largest ellipticity. This is due to the star forming regions at the end of the bar which contribute at shorter wavelengths. Resonances created by the bar are possibly responsible for the star forming ring in the central region. The diameter of the ring is found to be $3^{\prime \prime}$. Faint signatures of the ring are also seen in the ellipticity and the colour profiles. However, since the seeing was $\sim 1.5^{\prime \prime}$, imaging at a higher resolution is required to study the details of the ring.

8. Mrk 1002: This galaxy is classified as S0 by Mazzarella \& Balzano (1986) and as E1 by the Markarian catalog. The central contours, as seen in Fig. 1h, appear elliptical. An inspection of the direct images reveals that at about $6^{\prime \prime}$, the contours start deviating from ellipses and seem to give a faint indication of spiral arms. Beyond $12^{\prime \prime}$, they regain their elliptical nature. The position angle jumps abruptly between $3^{\prime \prime}$ and $10^{\prime \prime}$. This is accompanied by a sudden sharp increase in ellipticity from less than 0.1 in the central region to 0.3 at $10^{\prime \prime}$ (Fig. $2 \mathrm{~h}$ ). This jump can be attributed to the spiral arm like features. Between $5^{\prime \prime}$ and $10^{\prime \prime}$ there is a twisting of isophotes, accompanied by boxiness of the isophotes in this region, as seen from the negative values of the B4 coefficient in Fig. 7. We detect a S-shaped blue structure crossing the nucleus in the color maps. The residual maps also show this structure partly. The excess luminosity in this structure shows a one-to-one correspondence with the blue areas in the colour map. The colour maps and the structural features imply star formation in

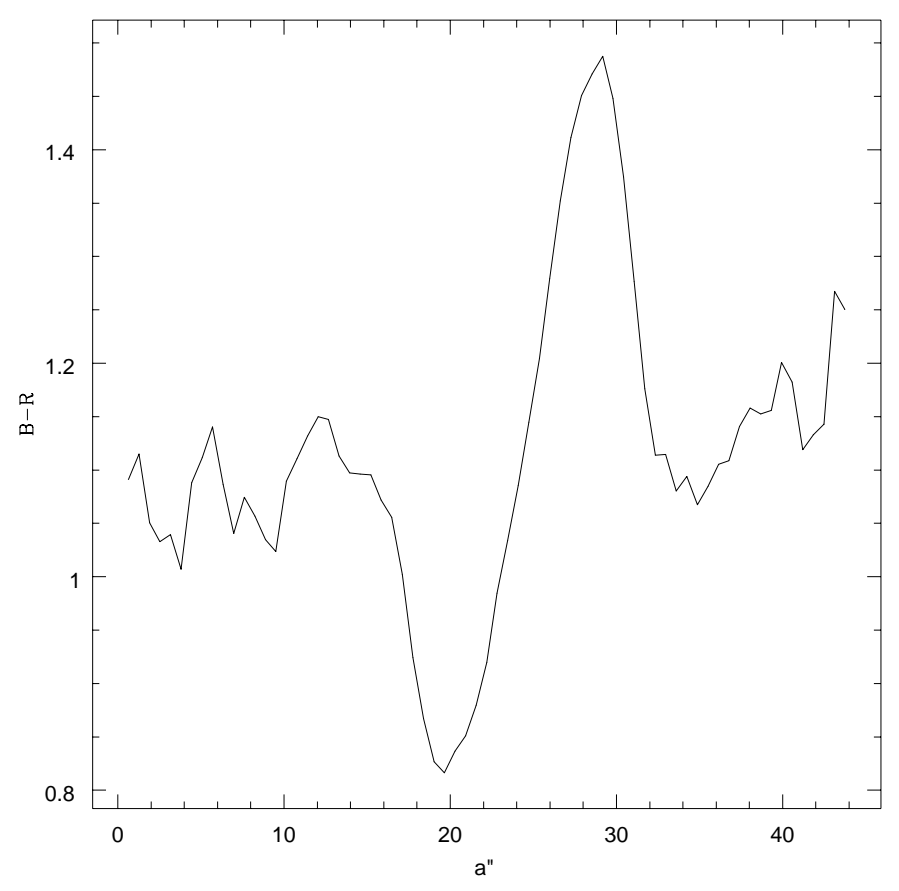

Fig. 5. Variation in $(B-R)$ along a cut through the nucleus and the dust lane in Mrk 449 at a position angle of $97^{\circ}$

certain regions of this galaxy. Pogge \& Eskridge (1993) have reported $\mathrm{H} \alpha$ emission in the nuclear as well as clumps of emission in the circumnuclear region in this galaxy. The rudimentary spiral pattern, the isophotal twists and the boxiness all suggest that this is a possible case of a merger wherein a gaseous disk was captured by this galaxy at some point. A similar spiral feature is found in the early type galaxy of the interacting pair AM $0327-285$ by de Mello et al. (1995) which they explain as arising due to the capture of a gaseous disk by the early type galaxy. However, because of the complex nature of the structure, a definitive answer can only be given with additional kinematical information on this galaxy.

9. Mrk 1308: This is a small nearby galaxy of S0 type extending about half an arcminute. It has a small linear companion located at $30^{\prime \prime}$ towards the west, which has been confirmed to be a physical neighbour by $6 \mathrm{~m}$ telescope spectroscopy (Doublier et al. 1997). The contours in the $B$ and $I$ filter bands appear smooth (see Fig. $1 \mathrm{j})$. However, we detect a very strong systematic twisting of isophotes in this galaxy (Fig. 2h). Though the ellipticity shows a small variation of less than 0.1 beyond the inner $3^{\prime \prime}$ there is a continuous variation in the position angle. Within the inner $10^{\prime \prime}$, the position angle changes by nearly $180^{\circ}$. Mrk 1308 exhibits strong isophotal twisting overall and non-concentric isophotes in the outer regions. The B4 coefficient oscillates around zero and shows no clear trends. Besides the blue nuclear region, there are no other features detectable in the colour maps. The $(B-V)$ colour for Mrk 1308 is 0.05 in the central regions and gets redder outwards, reaching a value 


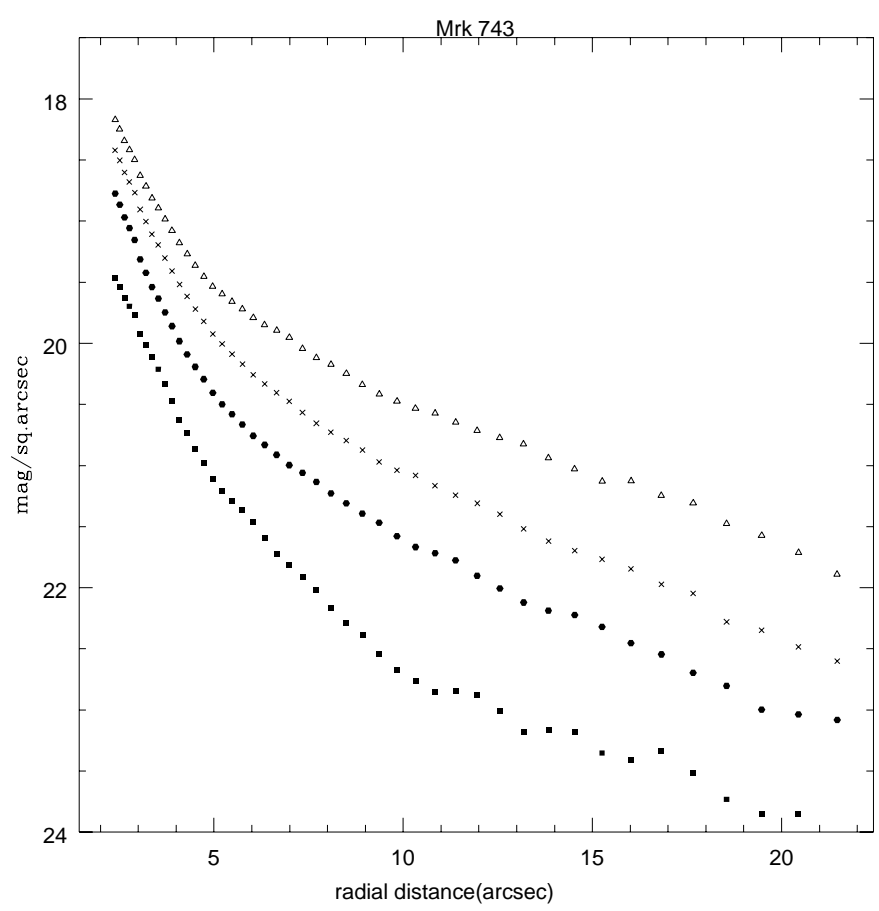

Fig. 6. Surface brightness distribution of Mrk 743 in $B$ (filled squares), $V$ (filled hexagons), $R$ (crosses) and $I$ (triangles)

of 0.7 near the periphery. The companion is a red object having a mean $(B-V)$ of 0.9 . The star formation activity is confined to the nuclear region. Radio imaging at $6 \mathrm{~cm}$. (Neff \& Hutchings 1992) shows that the emission is in the form of a ring-like structure of diameter $\approx 3^{\prime \prime}$. The residual image obtained after subtracting a smoothed image constructed using the isophotal analysis, reveals a bright nucleus and another blob to its north. The tidal interactions induced by the close companion could be responsible for the non-axisymmetric perturbations seen in the central part of Mrk 1308. The gas inflow to the nucleus and hence the star formation in the nucleus could arise as a result of these perturbations. These interactions seem to be the source of the starburst phenomenon in this galaxy.

10. Mrk 1379: is a VV object (Vorontsov-Velyaminov 1977), with nests of interacting objects. The irregular galaxy lying toward the eastern edge of Mrk 1379 was first masked out to prevent the results of ellipse fitting to be influenced by its luminosity. The position angle increases from $60^{\circ}$ to $75^{\circ}$ between $5^{\prime \prime}$ and $8^{\prime \prime}$ accompanied by a kink in the ellipticity profile between these points. We detect two peaks in the ellipticity profiles, the ellipticities of which are wavelength dependent. The first one corresponds to the blue structure lying at $13^{\prime \prime}$ to the west of the nucleus. Also, the local minima at $19^{\prime \prime}$ between the two maxima becomes more prominent at longer wavelengths. There is no appreciable variation in the position angle in these two regions. However, the surface brightness profiles have a small kink at this position in all the filters, the most prominent being in the $B$ band. In the inner $10^{\prime \prime}$ the blue

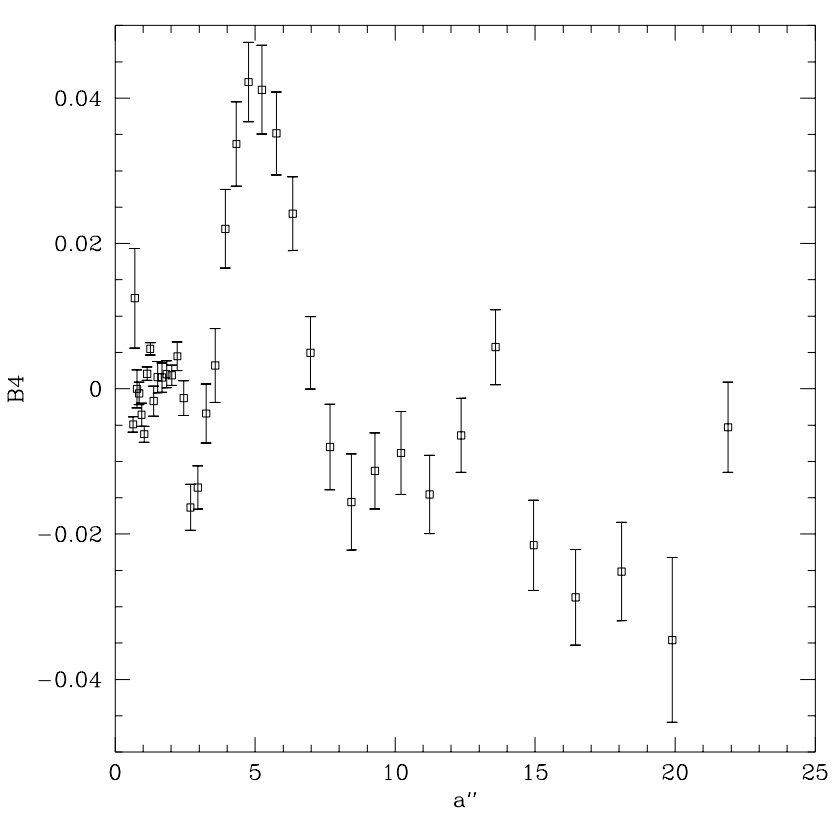

Fig. 7. The B4 coefficient as a function of the semi-major axis for Mrk 1002

isophotes are rounder. Between $10^{\prime \prime}$ and $15^{\prime \prime}$ the ellipticity profiles do not show any wavelength dependence. Beyond $15^{\prime \prime}$ the trend reverses and the $I$ band profiles are rounder. Beyond $30^{\prime \prime}$ the contribution to the blue light is predominantly from the spiral arms while the light distribution at longer wavelengths is smoother and in the form of a common envelope, which accounts for the wavelength dependent behaviour of the ellipticity profiles in this region. The $(B-I)$ image shows nuclear as well as extra nuclear star formation. Knots of star formation are seen in the nuclear region as well as along the western periphery of the galaxy at the point where the spiral arms start. In addition to this, global star formation is detected in both the companions lying to the east. The residual maps in the $B$ and the $I$ band appear different. Enhancements are seen in the nuclear region, in the blue knot to the west and along a curved spiral arm which appears to start from the nucleus. However, on inspection of the fine structure in the $I$ band, we clearly detect a short bar in the central region. We also detect spiral arms with two bright blobs connected to this inner linear structure.

\section{Decomposition parameters}

The decomposition of a galaxy profile into a bulge and a disk component requires the profile to be fitted by the sum of two empirical laws. The presence of structures like bars, dust lanes, lenses and rings render such a straightforward two component fit very difficult. The luminosity profiles of the program galaxies are complex in nature. 
A. Chitre and U.C. Joshi: Photometric studies of some starburst galaxies
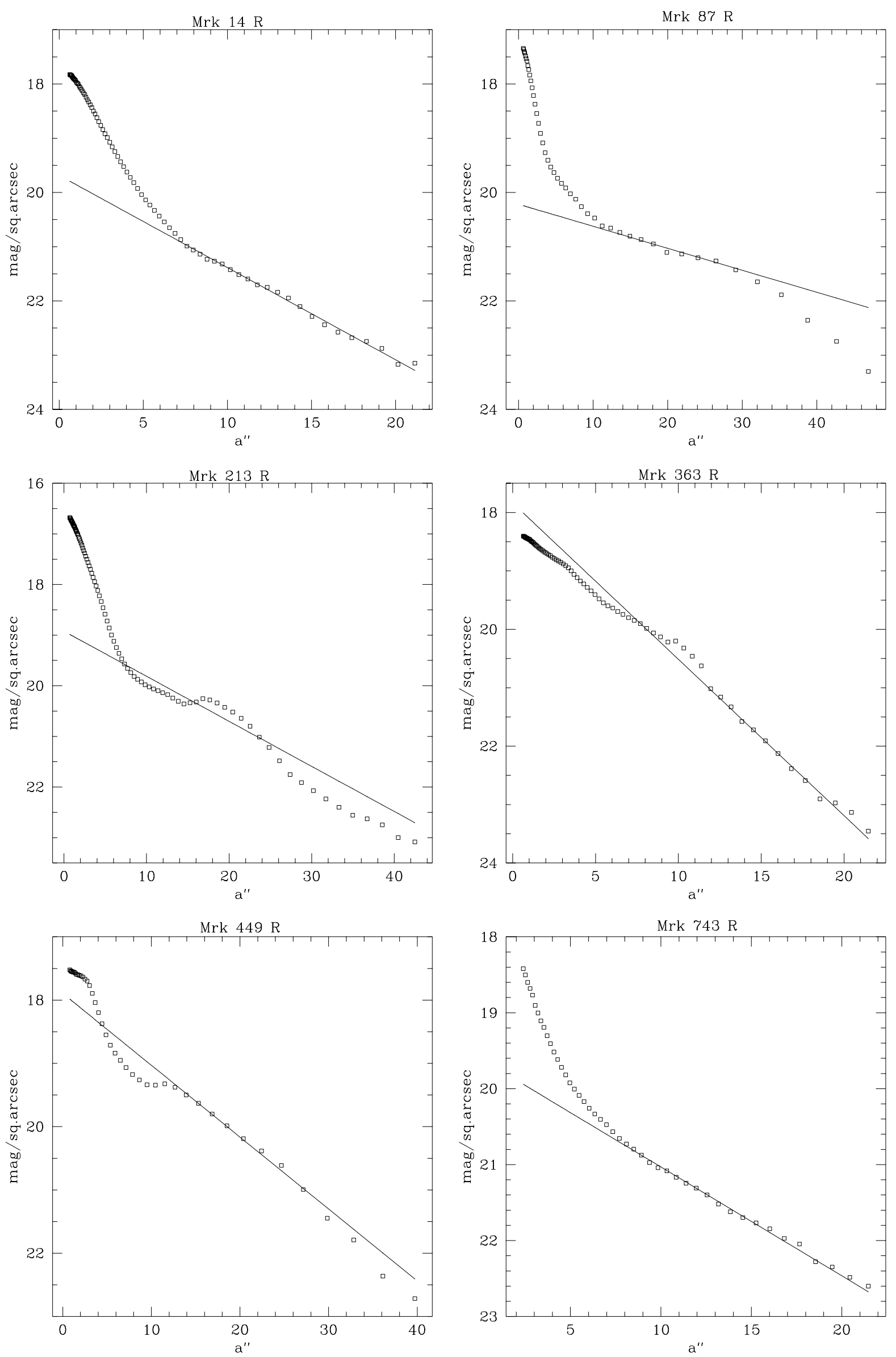

Fig. 8. a,b,c,d,e,f) Exponential fit to the $R$ bandpass luminosity profiles 

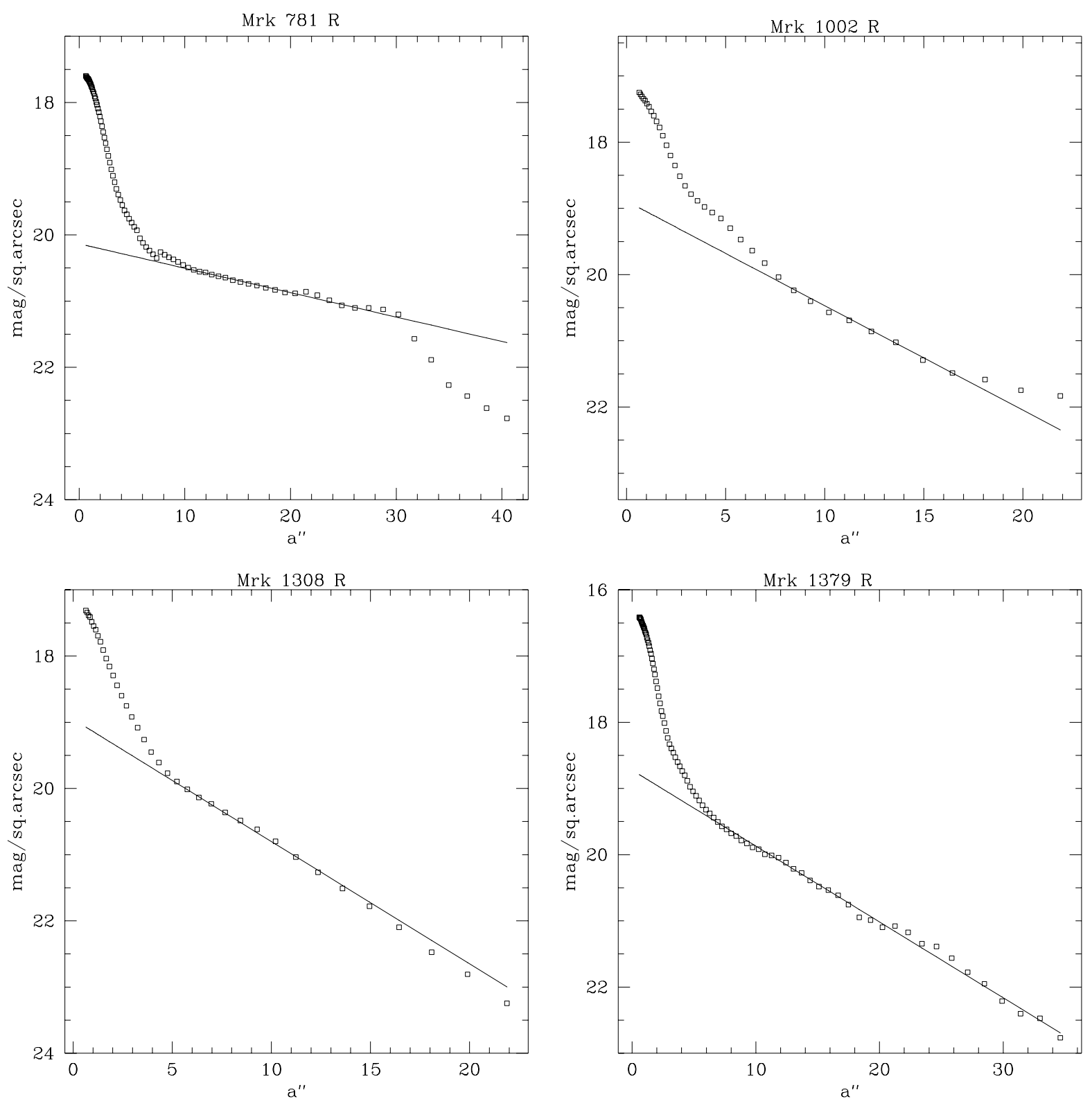

Fig. 8. g,h,i,j) continued

The burst of star formation in the nuclear region manifests itself as a sharp rise in intensity in this region. The burst luminosity completely dominates the light output in this region particularly and the estimation of a bulge component becomes very difficult. Hence we characterize the light distribution by parameters like the half-light radius, the central disk surface brightness and the disk scale lengths.

\subsection{Scale lengths and central disk surface brightness}

In the Markarian starburst galaxies, the luminosity profiles are complex in nature. In the inner region, the profile falls steeply up to about $10^{\prime \prime}$ where the light is completely

dominated by the burst component. The outer parts of the luminosity profile in most cases can be well described by an exponential scaling law viz.

$\mu(r)=\mu_{0}+1.086\left(\frac{r}{h}\right)$.

The outer exponential nature is also seen in case of dwarf ellipticals dwarf irregulars and HII galaxies (Telles 1995). This outer part is likely to represent the old underlying population of the parent galaxy. Structural properties like scale lengths and central disk surface brightnesses can be derived from these profiles. We fit a exponential law to the outer part of the profile down to where the signal falls to $3 \sigma$ of the background noise level. Figure 8 depicts the fits to the outer regions. Good fits to the observed profiles are obtained for the galaxies Mrk 14, Mrk 743, Mrk 1002, 


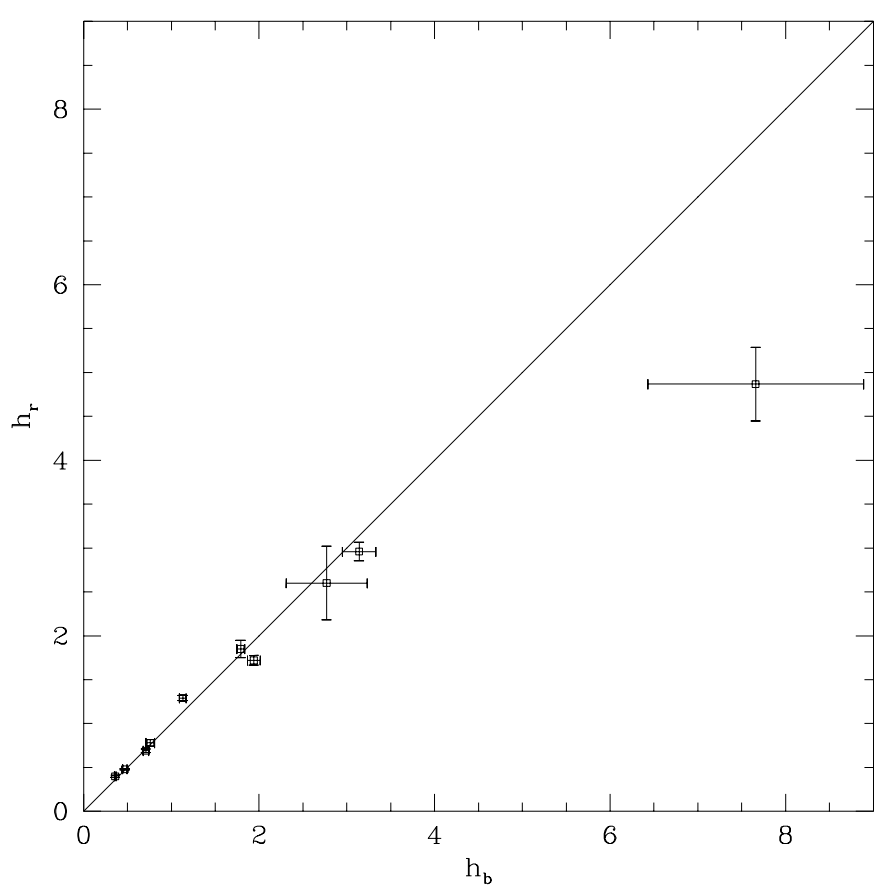

Fig. 9. Comparison of the scale lengths in $B\left(h_{\mathrm{b}}\right)$ and $R$ in $\left(h_{\mathrm{r}}\right)$. The points are the derived values and the solid line is the locus of $h_{\mathrm{b}}=h_{\mathrm{r}}$

Mrk 1308 and Mrk 1379. In case of Mrk 449, the luminosity profile shows a sharp dip due to the presence of the dust lane and hence the fitted values are higher than the observed values for surface brightness in this region. We have deliberately included a part of the bar into the range of fit for Mrk 213, Mrk 781 and Mrk 1379 since we found that excluding the bar region tends to overestimate the intensity in the inner regions of the galaxies. The results of exponential fits to each filter are tabulated in Table 4. A plot of the scale length in $B$ versus the scale length in $R$ (Fig. 9) indicates that the blue scale lengths are comparable to the red scale lengths in all the cases except for Mrk 87. The exponential law fails to fit very well in the outer regions for Mrk 87 and the departures seen could be a result of this improper fit. The total magnitudes $B_{\mathrm{T}}, V_{\mathrm{T}}$, $R_{\mathrm{T}}$ and $I_{\mathrm{T}}$ were derived by extrapolating the fitted disk to infinity and summing over the flux.

\subsection{Half-light radius}

The growth curve in each filter band was used to determine the half-light radius, $a_{\mathrm{e}}$ namely the radius within which half of the total light of the galaxy is contained.

$m_{\mathrm{hl}}=m_{\mathrm{T}}+0.7525$.

The values for the total magnitudes were taken from Table 3. to compute the half-light radii. The half-light radii derived for the sample galaxies in each of the filter

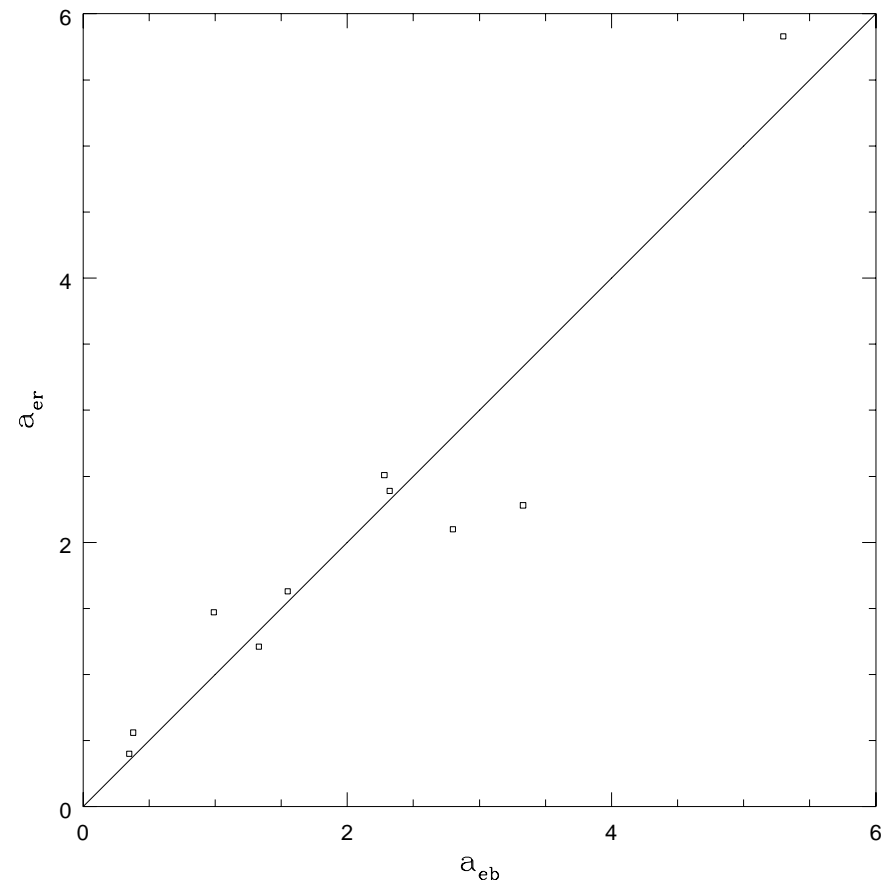

Fig. 10. Comparison of the half-light radius in $B\left(a_{\mathrm{eb}}\right)$ and in $R$ $\left(a_{\mathrm{er}}\right)$. The points denote the derived values and the solid line is the locus of $a_{\mathrm{eb}}=a_{\mathrm{er}}$

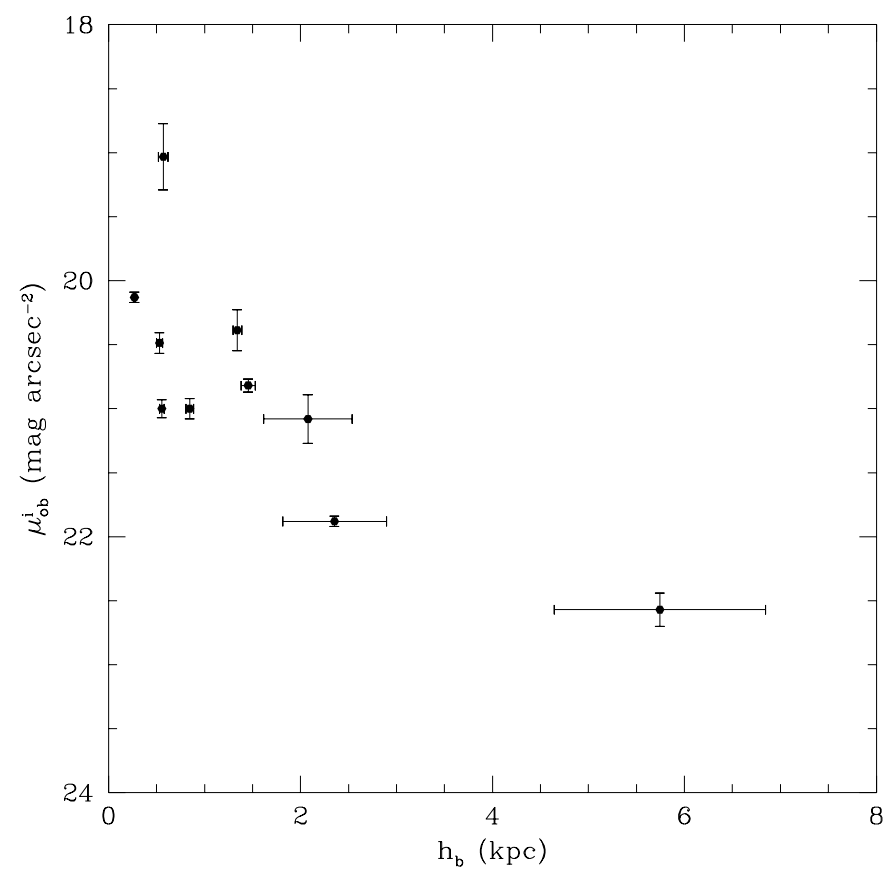

Fig. 11. Plots of the blue central disk surface brightness versus the blue scale length. For comparison with the values obtained by de Jong, the scale lengths have been transformed appropriately 
Table 4. Distribution of half-light radii $\left(a_{\mathrm{e}}\right)$, scale lengths $(h)$ and the central disk surface brightnesses $\left(\mu_{\mathrm{o}}\right)$. The subscripts $b, v$, $r, i$ denote the filters for which the values are presented. The half-light radii and scale lengths are in kpc, the surface brightness in $\mathrm{mag} / \mathrm{sq}$ arcsec and the range of fit in arcsec. The first row gives the derived values and the second row gives the errors on the corresponding quantities

\begin{tabular}{|c|c|c|c|c|c|c|c|c|c|c|c|c|c|}
\hline Galaxy & $a_{\mathrm{eb}}$ & $a_{\mathrm{ev}}$ & $a_{\mathrm{er}}$ & $a_{\mathrm{ei}}$ & $h_{\mathrm{b}}$ & $h_{\mathrm{v}}$ & $h_{\mathrm{r}}$ & $h_{\mathrm{i}}$ & $\mu_{\mathrm{ob}}$ & $\mu_{\mathrm{ov}}$ & $\mu_{\mathrm{or}}$ & $\mu_{\mathrm{oi}}$ & range of fit \\
\hline \multirow[t]{2}{*}{14} & 0.99 & 1.34 & 1.47 & 1.47 & 1.13 & 1.37 & 1.29 & 1.27 & 20.76 & 20.39 & 19.68 & 19.10 & $7-21$ \\
\hline & & & & & 0.04 & 0.02 & 0.03 & 0.04 & 0.08 & 0.03 & 0.04 & 0.07 & \\
\hline \multirow[t]{2}{*}{87} & 2.8 & 2.4 & 2.1 & 2.5 & 7.66 & 5.89 & 4.87 & 4.10 & 21.92 & 21.04 & 20.22 & 19.39 & $14-31$ \\
\hline & & & & & 1.23 & 1.22 & 0.42 & 0.22 & 0.07 & 0.14 & 0.07 & 0.05 & \\
\hline \multirow{2}{*}{213} & 3.38 & 2.75 & 2.28 & 3.03 & 2.77 & 2.75 & 2.60 & 2.74 & 20.09 & 19.61 & 19.01 & 18.54 & $13-42$ \\
\hline & & & & & 0.46 & 0.35 & 0.42 & 0.32 & 0.19 & 0.15 & 0.19 & 0.15 & \\
\hline \multirow[t]{2}{*}{363} & 1.55 & 1.59 & 1.63 & 1.84 & 0.76 & 0.82 & 0.78 & 1.02 & 18.72 & 18.55 & 17.82 & 18.27 & $14-21$ \\
\hline & & & & & 0.05 & 0.04 & 0.04 & 0.03 & 0.26 & 0.10 & 0.23 & 0.19 & \\
\hline \multirow[t]{2}{*}{449} & 1.33 & 1.33 & 1.21 & 1.46 & 0.71 & 0.73 & 0.69 & 0.66 & 19.18 & 18.49 & 17.89 & 17.19 & $14-40$ \\
\hline & & & & & 0.032 & 0.025 & 0.019 & 0.023 & 0.08 & 0.06 & 0.05 & 0.07 & \\
\hline \multirow[t]{2}{*}{743} & 0.35 & 0.49 & 0.40 & 0.54 & 0.74 & 0.45 & 0.48 & 0.57 & 21.10 & 20.02 & 19.60 & 19.25 & $7-21$ \\
\hline & & & & & 0.024 & 0.007 & 0.006 & 0.011 & 0.07 & 0.06 & 0.04 & 0.03 & \\
\hline \multirow[t]{2}{*}{781} & 5.30 & 6.00 & 5.83 & 5.85 & 3.14 & 3.07 & 2.96 & 2.47 & 21.44 & 20.71 & 20.11 & 19.32 & $10-40$ \\
\hline & & & & & 0.54 & 0.21 & 0.18 & 0.14 & 0.04 & 0.03 & 0.03 & 0.03 & \\
\hline \multirow[t]{2}{*}{1002} & 2.28 & 2.28 & 2.51 & 2.51 & 1.79 & 1.78 & 1.85 & 1.79 & 20.06 & 19.39 & 18.86 & 18.38 & $7-22$ \\
\hline & & & & & 0.045 & 0.137 & 0.098 & 0.090 & 0.16 & 0.11 & 0.10 & 0.11 & \\
\hline \multirow[t]{2}{*}{1308} & 0.38 & 0.53 & 0.56 & 0.65 & 0.36 & 0.39 & 0.40 & 0.43 & 20.02 & 19.44 & 18.95 & 18.45 & $7-21$ \\
\hline & & & & & 0.008 & 0.005 & 0.012 & 0.011 & 0.04 & 0.03 & 0.04 & 0.03 & \\
\hline \multirow[t]{2}{*}{1379} & 2.32 & 2.63 & 2.39 & 2.83 & 1.94 & 1.81 & 1.72 & 1.66 & 20.17 & 19.37 & 18.72 & 17.96 & $11-35$ \\
\hline & & & & & 0.074 & 0.054 & 0.056 & 0.047 & 0.05 & 0.04 & 0.05 & 0.04 & \\
\hline
\end{tabular}

bands are presented in Table 4. Figure 10 shows the plot of the half light radius in $B$ versus the half-light radius in $R$. The plot does not show any clear trends, however there are indications of $a_{\mathrm{eb}}$ being smaller than $a_{\mathrm{er}}$ in most cases. This suggests that the blue light is more centrally concentrated then the red light in most of these objects within the surface brightness limits reached by our data. This is to be expected in case of starburst galaxies as the starburst activity is nuclear or circumnuclear in most of the galaxies.

\section{Comparison with other samples}

The derived parameters viz. the scale lengths and the central disk surface brightnesses were computed with those derived by de Jong (1996) for normal spiral galaxies. The plot of the blue scale length versus the corrected central disk brightness (Fig. 11) shows that the Markarian starburst galaxies in our sample have scale lengths less than about $2 \mathrm{kpc}$ in the $B$ band. These are shorter compared to those derived for normal spirals by de Jong using a bulge-disk decomposition. The central disk surface brightness is also brighter than that observed in normal spirals by other workers. Bothun et al. (1989) report a mean value of $2 \mathrm{kpc}$ for the scale lengths derived using "marking the disk" based on the study of the Wasilewski sample of emission-line galaxies in the Gunn $R$ band. They also find the mean central surface brightness of exponential disks to be about 1.5 mag brighter than that for normal spirals in Gunn $R$. However, Courteau (1996) cautions against the comparison of scale lengths derived by different workers due to the subjective nature of the measurements. He argues that "marking the disk" approach would lead to smaller scale lengths over the bulge-disk decomposition fits. In the $(B / D)$ decompositions, the inclusion of a bulge reduces the amount of light contributed by the inner disk and thus leads to a shallower slope for the disk profiles. Knapen \& van der Kruit (1991) find discrepancies up to a factor of two in the scale lengths measured by various workers.

\section{Properties of the burst region}

Huchra (1977) was the first to propose that the starburst galaxy can be thought of as made up of stellar populations of two ages: the underlying galaxy which is the old galaxy, superimposed on which is the young burst component. To estimate the burst component, the contribution of the underlying galaxy has to be subtracted. This is not an easy task. Aperture photometry of the region in which the young population is present gives the total intensity in that region. The contribution of the galaxy can be subtracted by estimating the galaxy from an annular region around the burst region. However, this approach suffers from a major drawback. The galaxy contribution estimated by this method gives the underlying galaxy value outside the region and not at the position of the burst. Such an approach would underestimate the 
Table 5. Colours of the burst component

\begin{tabular}{llll}
\hline Galaxy & $B-V$ & $V-R$ & $R-I$ \\
\hline Mrk 14 & 0.33 & 0.36 & 0.32 \\
Mrk 87 & 0.54 & 0.63 & 0.55 \\
Mrk 213 & 0.63 & 0.63 & 0.42 \\
Mrk 363 & - & - & - \\
Mrk 449 & 0.09 & 0.16 & 0.111 \\
Mrk 743 & 0.38 & 0.32 & 0.22 \\
Mrk 781 & 0.67 & 0.48 & 0.38 \\
Mrk 1002 & 0.37 & 0.50 & 0.04 \\
Mrk 1308 & -0.04 & 0.29 & 0.14 \\
Mrk 1379 & 0.36 & 0.47 & - \\
\hline
\end{tabular}

galaxy contribution especially when the underlying galaxy luminosity profile is an exponential disk as described in the previous section. This would lead to erroneous values for the colour of the burst. To overcome this difficulty, we use the disk component estimated in the last section to derive the burst values. The exponential disk is extrapolated right up to the central regions. We construct a disk model of the galaxy. This is then subtracted from the galaxy. The residual intensity in the central region gives us the contribution of the burst component. This approach is used only for the central region in each galaxy since nonaxisymmetric structures like bars can contribute to the residual in the outer regions. The burst colours derived after subtracting the disk component as described above are presented in Table 5. Mrk 363 shows globally enhanced star formation. This makes it impossible to separate the young and the old stellar components in this galaxy and hence estimate the burst colours from the results of the disk fitting.

\section{Conclusions}

The starburst activity is seen to be hosted by galaxies of nearly all morphological types. In case of elliptical or S0 galaxies in the sample, strong isophotal twisting has been detected, accompanied by boxiness in certain cases. Mrk 1002, Mrk 14 and Mrk 1308 all show strong isophotal twists. In addition, boxiness is detected in Mrk 1002 and Mrk 14. Fine structure like rudimentary spiral structure is observed in Mrk 1002. Nuclear bars have been detected in Mrk 213 and Mrk 1379. Tidal interactions, mergers or accretion of material are the most likely triggers for the starburst phenomena observed in S0s and elliptical like galaxies in the sample. The spirals in the sample show the presence of a bar responsible for fueling the starburst or the presence of a bar-like structure in the central nuclear region as in the case of Mrk 213.

Acknowledgements. This work was supported by the Department of Space, Government of India. The authors are thankful to Mr. A.B. Shah, Mr. N.M. Vadher and Mr. Shashikiran Ganesh for technical support. We are grateful to the referee, Dr.Wozniak for valuable suggestions.

\section{References}

Arkhipova V.P., 1982, Sov. A. J. 26, 129

Arsenault R., 1989, A\&A 217, 66

Balzano V., 1983, ApJ 268, 602

Barth C.S., Coziol R., Demers S., 1995, MNRAS 276, 1224

Bothun R.D., Halpern J.P., Lonsdale C.J., et al., 1989, ApJS 70, 271

Bender R., Möllenhoff C., 1987, A\&A 177, 71

Burstein D., Krumm N., Salpeter E.E., 1987, AJ 94, 883

Buta R., 1986, ApJS 61, 631

Combes F., 1987, in Starbursts and Galaxy Evolution, Thuan T.X., Montmerle T. \& Tran Thanh Van J. (eds.). Éditions Frontières, Gif-sur-Yvette, p. 325

Combes F., Dupraz C., Gerin M., 1990, in Dynamics and Interactions of Galaxies, Wielen R. (ed.). Springer-Verlag, Heidelberg, p. 205

Courteau S., 1996, ApJS 103, 363

de Jong R.S., 1996, A\&A 313, 45

de Mello D.F., Keel W.C., Sulentic J.W., et al., 1995, A\&A 297,331

Doublier V., Comte G., Petrosian A., et al., 1997, A\&AS 124, 405

Garcia-Barreto J.A., Franco J., Carrillo R., et al., 1996, Rev. Mex. Astron. Astrofis. 32, p. 89

Geller M.J., Huchra J.P., 1983, ApJS 52, 61

Griersmith D., 1980, AJ 85, 789

Griersmith D., Hyland A.R., Jones B., 1982, AJ 87, 1106

Hawarden T.G., Longmore A.J., Tritton S.B., et al., 1981, MNRAS 196, 747

Hernquist L., 1992, ApJ 400, 460

Hernquist L., 1993, ApJ 409, 548

Huchra J.P., 1977a, ApJS 35, 171

Huchra J.P., 1977b, ApJ 217, 926

Hamuy M., Maza J., 1987, A\&AS 68, 383

Hunter D.A., Gallagher J.S.III., 1986, PASP 98, 5

Jedrejewski R.I., 1987, MNRAS 226, 747

Jungwiert B., Combes F., Axon D.J., 1997, A\&AS 125, 479

Keel W.C., van Soest E.T.M., 1992, A\&AS 94, 553

Kenney J.D.P., Koopmann R.A., Rubin V.C., et al., 1996, AJ 111,152

Knapen J.H., Van Der Kruit P.C., 1991, A\&A 248, 57

Landolt A.U., 1992, AJ 104, 340

Larson R.B., Tinsley B.M., 1978, ApJ 219, 46

Markarian B.E., Lipovetskii V.A., Stepanian D.A., 1979, Afz 15,549

Mazzarella J.M., Balzano V.A., 1986, ApJS 62, 751

Mazzarella J.M., Boroson T.A., 1993, ApJS 85, 27

Mc Clure R.D., van den Bergh S., 1968, AJ 73, 1008

Neff S.G., Hutchings J.B., 1992, AJ 104, 1

Nieto J.L., Bender R., 1989, A\&A 215, 266

Pogge R.W., Eskridge P.B., 1993, AJ 106, 1405

Prugniel Ph., Heraudeau Ph., 1998, A\&AS 128, 299

Schweizer F., Seitzer P., 1988, ApJ 328, 88

Schweizer F., Seitzer P., 1992, ApJ 104, 1039

Scoville N.Z., Hersh K., 1979, ApJ 229, 578

Telles J.E., 1995 , Ph.D. thesis, University of Cambridge

Terlevich R., 1992, in: Relationships between Active Galactic Nuclei and Starburst Galaxies, ASP Conf. Ser. 31, Alexei V. Filippenko. (eds.), p. 133

Tully R.B., 1988, Nearby Galaxies Catalog. Cambridge University Press 
van Driel W., van Woerden H., 1991, A\&A 243, 71 van Moorsel G., 1988, A\&A 202, 59

Vorontsov-Velyaminov B.A., 1977, A\&AS 28, 1

Weedman D.W., 1973, ApJ 183, 29

Wozniak H., Pierce M.J., 1991, A\&AS 88, 325
Wozniak H., Friedli D., Martinet L., et al., 1995, A\&AS 111, 115

Wrobel J.M., Heeschen D.S., 1988, ApJ 335, 677

Wrobel J.M., Heeschen D.S., 1991, AJ 101, 148 